\title{
Hurwicz Expected Utility and Subjective Sources ${ }^{\dagger}$
}

\author{
Faruk Gul \\ and \\ Wolfgang Pesendorfer \\ Princeton University
}

April 2015

\begin{abstract}
In experiments, subjects are often not indifferent among all sources of uncertainty; between two prospects yielding the same distribution of monetary rewards, they may strictly prefer one over the other. We formulate a special case of $\alpha$-maxmin expected utility theory in a Savage setting, show that every decision maker perceives multiple subjective sources, and that source-utilities are rank dependent expected utility. A power series identifies each source, measures source-uncertainty, and determines the agent's sourcespecific risk attitude. Subjective sources connect Ellsberg-paradox behavior with source preference and with Allais-paradox behavior.

$\dagger$ This research was supported by a grant from the National Science Foundation (Grant number: SES-0820101). We are grateful to Adriano Basso and Jay Lu for many insightful comments and suggestions. Authors' affiliation and address: Faruk Gul, Deparment of Economics, Princeton University; fgul@princeton.edu; Wolfgang Pesendorfer, Department of Economics, Princeton University; pesendor@princeton.edu
\end{abstract}

C 2015. This manuscript version is made available under the Elsevier user license 


\section{Introduction}

In experiments, many subjects exhibit behavior inconsistent with subjective expected utility theory. These subjects either violate the independence axiom (Allais-style evidence $^{1}$ ) or choose among bets in a way that is inconsistent with a subjective probability (Ellsberg-style evidence ${ }^{2}$ ); or they reverse their ranking of risky prospects when the source of the uncertainty changes (source-preference evidence ${ }^{3}$ ). Numerous theoretical papers deal with the first ${ }^{4}$ or the second ${ }^{5}$ phenomenon and some deal with the third. ${ }^{6}$ In this paper, we show that a special case of a standard model, $\alpha$-maxmin expected utility $(\alpha-\mathrm{MEU})$ theory, can address all three.

We start with a preference over Savage acts with monetary prizes and give axioms that are equivalent to the following representation: the decision maker has a set of priors $\Pi_{\mu}$ such that the utility $W(f)$ of a real-valued act $f$ is

$$
W(f)=\alpha \min _{\pi \in \Pi_{\mu}} \int v \circ f d \pi+(1-\alpha) \max _{\pi \in \Pi_{\mu}} \int v \circ f d \pi
$$

where $v$ is the utility index and $\alpha \in[0,1]$ is the parameter of uncertainty aversion. The set $\Pi_{\mu}$ consists of all possible extensions of $\mu$; that is, $\Pi_{\mu}$ is the set of all priors that agree with the prior $\mu$ on its domain (the $\sigma$-algebra $\mathcal{E}_{\mu}$ ). We refer to the elements of $\mathcal{E}_{\mu}$ as $i d e a l$ sets. Hence, the representation above suggests that the agent is completely ignorant about probabilities beyond the bounds implied by $\mu$. We call our model Hurwicz expected utility theory (HEU) since it combines subjective expected utility theory with the Hurwicz $\alpha$-criterion (Hurwicz (1951)).

HEU preferences are the subset of $\alpha$-MEU preferences identified by the restriction described above on the set of priors. By design, $\alpha$-MEU addresses Ellsberg-style evidence and, as we show in Gul and Pesendorfer (2014), the restriction on the set of priors does not diminish HEU's ability to a accommodate this evidence. In particular, HEU is flexible

\footnotetext{
1 Allais (1953), Kahnemann and Tversky (1979)

2 Ellsberg (1961)

3 French and Poterba (1991), Heath and Tversky (1991)

4 See Starmer (2000) for a survey of the theoretical and experimental literature on Allais-style evidence.

5 See Gilboa and Marinacci (2013) for a survey of the theoretical literature that addresses Ellsberg-style evidence.

6 Among the theoretical multi-source models are Klibanoff, Marinacci and Mukerji (2005), Nau (2006), Ergin and Gul (2009), Chew and Sagi (2008), Abdellaoui et al. (2011).
} 
enough to accommodate all versions of the 2-prize Ellsberg paradox. ${ }^{7}$ Here, we show how HEU can address source-preference and Allais-style evidence as well. The latter confronts agents with lotteries (over prizes) instead of Savage acts. The former considers situations in which agents reduce acts to lotteries as long as all the relevant uncertainty depends on a single "source." To analyze both of these phenomena together, we will identify circumstances when HEU agents are probabilistically sophisticated (Machina and Schmeidler (1992)); that is, when they use a prior to reduce acts to lotteries. We call such a prior (and the associated $\sigma$-algebra of events) a source.

Consider a group of HEU agents with identical sets of priors $\Pi_{\mu}$ but possibly different uncertainty attitudes $(\alpha, v)$. A source for these agents is a prior $\pi$ such that each agent in the group ranks bets on source events in accordance with the prior $\pi$. For example, suppose our agents must rank bets on an "ambiguous" Ellsberg urn containing different colored balls $\left(c_{1}, \ldots, c_{n}\right)$ of unknown proportion. Quite plausibly, every agent in the group is indifferent between a bet on $c_{i}$ or $c_{j}$ and, more generally, ranks bets on $A \subset\left\{c_{1}, \ldots, c_{n}\right\}$ and $B \subset\left\{c_{1}, \ldots, c_{n}\right\}$ simply according to the cardinality of the two events. ${ }^{8}$ In that case, the uniform prior on the colors of the urn is a source. ${ }^{9}$ In Proposition 2 (and Proposition $2 \mathrm{w}$ ), we show that HEU admits a rich collection of sources and characterize them. Sources are subjective in the sense that they are derived from the agents' rankings of Savage-acts over monetary prizes. Hence, in contrast to the urn example above, we do not assume coherent preferences over exogenously given collections of events; rather, we show that if preferences admit an HEU representation, there are multiple subjective sources.

A decision maker may consider the S\&P500, the Democratic vote share in the next election, or draws of a roulette wheel a source. In each of these cases, the agent's betting preference might plausibly be compatible with a subjective probability. ${ }^{10}$ Yet, as many

7 In Gul and Pesendorfer (2014), we analyze expected uncertain utility theory, a more general model. However, for the special case of two prizes, the more general model analyzed in Gul and Pesendorfer (2014) and HEU coincide.

8 Notice that this agreement over the ranking of bets only holds when we restrict to bets on the ambiguous urn. It breaks down when comparing a bet on the ambiguous urn with a bet on an urn with known proportions of colored balls. In that case, the uncertainty attitude $\alpha$ will affect agents' rankings of bets.

9 Our definition of a source adds richness and continuity requirements. Specifically, the prior must be non-atomic and, thus, the source must contain a rich collection of events.

10 Abdellaoui, Baillon, Placido and Wakker (2011) report experimental evidence for several sources of uncertainty, among them the French stock index, the temperature in Paris and two Ellsberg-style urns. For all but one of their sources, the authors cannot reject the hypothesis that subjects' betting preferences are compatible with a subjective probability. They estimate subjects' source-specific uncertainty attitudes and find that they vary with the source. 
experiments have found, the preference may fail to be compatible with a probability if bets depend on two or more of those sources. Tversky and Fox (1995) and Tversky and Wakker (1995) coin the term source preference to describe this phenomenon. Two main conclusions emerge from experimental research on source-preference: first, subjects have a source preference not only when choosing between completely vague and probabilistically described sources (Chipman (1960), Curley and Yates (1989)) but also when choosing between sources with somewhat more or somewhat less vague specification or between sources about which they consider themselves to be more or less informed (Heath and Tversky (1991)). Second, subjects tend to reverse their preference for less vague or more familiar sources when the probability of winning is small. When choosing between bets that have long-odds, subjects prefer vagueness (Curley and Yates (1989)) or sources about which they know less (Heath and Tversky (1991)).

HEU agents with an intermediate level of uncertainty aversion exhibit the patterns of behavior found in the experimental literature on source preference. A power series characterizes each source and measures its uncertainty (Proposition 4). While maximally uncertainty averse agents $(\alpha=1)$ always prefer bets on less uncertain sources, agents with an intermediate level of uncertainty aversion $(0<\alpha<1)$ reverse this preference when the probability of winning is low (Proposition 5). Hence, with $\alpha=1$, HEU theory accommodates the extreme uncertainty aversion suggested by Ellsberg's original experiment but when $0<\alpha<1$, HEU theory yields the more nuanced findings of subsequent empirical and experimental work.

Allais-style experiments confront subjects with lotteries; that is, acts that depend on a roulette wheel, on the draws of a card from a deck, or on some other objective randomization device. ${ }^{11}$ In a subjective model such as ours, the randomization devices in Allais-style experiments are a source like any other; randomization devices need not yield the least uncertain or most preferred source nor do all randomization devices necessarily yield the same source. Indeed, Heath and Tversky (1991) provide experimental evidence showing

\footnotetext{
11 Of course, some experiments simply state probabilities without specifying a randomization device. We interpret this as a situation in which one of many possible randomization devices will be used to determine outcomes but the agent does not know which.
} 
that agents may not favor sources based on randomization devices. ${ }^{12}$ In addition, the experimental literature has found that measured risk attitudes vary with the experimental technique used to measure those attitudes. Thus, subjects differentiate among seemingly objective sources. ${ }^{13}$

A (monetary) act that depends on a single source is a random variable and its cumulative distribution function is all that matters for an HEU-maximizer's utility. However, the function that maps these cumulatives to utilities is source-dependent. We refer to these functions as source utilities and show (Proposition 6) that they belong to the class of rank dependent expected utilities (RDEU, Quiggin (1982)). RDEU has two parameters, a utility index (analogous to the von Neumann Morgenstern utility index) and a probability transformation function. The utility index is the same for all sources but the probability transformation function depends on the source's power series. In many sources, the resulting probability transformation function has an inverted-S shape, as typically used in applications that address Allais-style evidence. Kahnemann and Tversky (1979), Tversky and Kahneman (1992), Camerer and Ho, (1994), Wu and Gonzalez, (1996), Prelec (1998) and Abdellaoui (1998) provide evidence in favor of the inverted S-shape.

Risk aversion in a source depends on the agent's risk and uncertainty attitude $(\alpha, v)$ ) but also on how uncertain she perceives the source to be. The latter effect is measured by the source polynomial. For a given source polynomial, the parameters $\alpha$ and $v$ affect risk attitudes in a systematic way: a more concave utility index implies more risk aversion while greater uncertainty aversion (greater $\alpha$ ) implies greater first order risk aversion; that is, a greater tendency to purchase full insurance at actuarially unfair odds. HEU agents perceive a range of sources of varying degrees of uncertainty and, therefore, we expect measured risk aversion to be context dependent, just as experimental and field studies find. ${ }^{14}$ At the same time, HEU agents have stable, context-independent uncertainty attitudes as measured by $v$ and $\alpha$ and, therefore, we expect the riskiness of agent's choices to be correlated across sources, as found in the empirical work of Einav et al. (2012).

\footnotetext{
12 Specifically, Heath and Tversky (1991) show that subjects tend to prefer familiar "subjective" bets over equally likely bets on the outcome of a randomization device if these bets have a high probability of success.

13 See, for example, Dave et al (2010).

14 See, for example, Isaac and James (2000) and Einav et al. (2012).
} 


\subsection{Relation to the Literature}

The HEU representation theorem is a special case of the representation theorem of expected uncertain utility theory (Gul and Pesendorfer (2014)). We obtain HEU by strengthening one of the axioms of the earlier representation. Zhang's (2002) model of Choquet expected utility is a special case of HEU and corresponds to the case in which $\alpha=1$. The theoretical literature contains relatively few multi-source models. Klibanoff, Marinacci and Mukerji (2005) and Ergin and Gul (2009) consider preferences that permit two distinct sources. These papers focus on the relationship between the two-source model and compound lotteries. Chew and Sagi (2008) define sources in a general Savage-style model which they call small worlds. They give conditions under which a lottery preference characterizes the agent's behavior in any source and provide examples of preferences with multiple sources. Nau (2006) provides a more general notion of source and sourcedependent risk attitude that permits two such sources with state-dependent preferences. Abdellaoui, Baillon, Placido and Wakker (2011) study source specific lottery preferences and estimate source-specific RDEU utility functions. Our model exhibits related source utilities and, in addition, provides a utility function for arbitrary multi-source acts.

\section{The Model}

The non-degenerate interval $X=[l, m]$ is the set of prizes, $\Omega$ is the state space, and elements of $\mathcal{F}=\{f: \Omega \rightarrow X, f(\Omega)$ finite $\}$, the set of simple Savage acts, are the choice objects. Our primitive is $\succeq$, a binary relation on $\mathcal{F}$.

To simplify the exposition, we use the following notational convention. For any property $\mathrm{P}$, let $\{\mathrm{P}\}$ denote the set of all $\omega \in \Omega$ at which $\mathrm{P}$ holds. For example, $\{f>$ $g\}=\{\omega \mid f(\omega)>g(\omega)\}$. For $\{\mathrm{P}\}=\Omega$, we simply write $\mathrm{P}$; that is, $f \in[x, y]$ means $\{\omega \mid f(\omega) \in[x, y]\}=\Omega$. We identify $x \in X$ with the constant act $f=x$. We write $f A g$ for the act that agrees with $f$ on $A$ and with $g$ on $A^{c}$, the complement of $A$.

A prior is a countably additive, complete, and non-atomic probability measure on some $\sigma$-algebra of subsets of $\Omega$. Let $\Pi$ denote the set of all priors and, for $\pi \in \Pi$, let $\mathcal{E}_{\pi}$ be the $\sigma$-algebra of $\pi$. The prior $\pi \in \Pi$ is an extension of $\mu \in \Pi$ if $\mathcal{E}_{\mu} \subset \mathcal{E}_{\pi}$ and $\pi(E)=\mu(E)$ for all $E \in \mathcal{E}_{\mu}$. Let $\Pi_{\mu}$ be the priors that are extensions of $\mu$. A utility index 
is a continuous, strictly increasing function $v: X \rightarrow \mathbb{R}$. The function $W: \mathcal{F} \rightarrow \mathbb{R}$ is a Hurwicz expected utility (HEU) if there is a prior $\mu$, a utility index $v$ and $\alpha \in[0,1]$ such that

$$
W(f)=\alpha \min _{\pi \in \Pi_{\mu}} \int v \circ f d \pi+(1-\alpha) \max _{\pi \in \Pi_{\mu}} \int v \circ f d \pi
$$

The proof of Proposition 1 in the appendix shows that (1) is well-defined; that is, the minimum and the maximum above are attained. The $\sigma$-algebra $\mathcal{E}_{\mu}$ consists of the events the decision maker perceives to be least uncertain. If the act $f$ is $\mathcal{E}_{\mu}$-measurable, then $W(f)=\int v \circ f d \mu$ and HEU reduces to subjective expected utility. To evaluate acts that are not $\mathcal{E}_{\mu}$-measurable, the decision maker uses a Hurwicz-criterion (Hurwicz (1951)) applied to $\Pi_{\mu}$, the extensions of $\mu$.

Proposition 1, below, is a representation theorem for HEU. To state the axioms for that result, we need the following definitions. The event $A$ is null if $f A h \sim g A h$ for all $f, g, h \in \mathcal{F}$. The event $E$ is ideal if for all acts $f, g, h$ and $h^{\prime}$

$$
[f E h \succeq g E h \text { and } h E f \succeq h E g] \text { implies }\left[f E h^{\prime} \succeq g E h^{\prime} \text { and } h^{\prime} E f \succeq h^{\prime} E g\right]
$$

Savage's sure thing principle (P2) is the requirement that every event is ideal. In HEU, ideal events represent the least uncertain events (corresponding to the events $\mathcal{E}_{\mu}$ in the representation) and the agent uses them to quantify the uncertainty of other, non-ideal, events. An event $D$ is diffuse if

$$
E \cap D \neq \emptyset \neq E \cap D^{c}
$$

for every non-null ideal event $E$. Diffuse events are the most uncertain events since they (and their complements) contain no (non-null) ideal events.

Let $\mathcal{E}$ be the set of all ideal events and $\mathcal{D}$ be the set of all diffuse events. We write $E, E^{\prime}, E_{i}$ for elements of $\mathcal{E}$ and $D, D^{\prime}, D_{i}$ etc. for elements of $\mathcal{D}$. Finally, let $\mathcal{F}^{e} \subset \mathcal{F}$ be the acts such that $f^{-1}(x) \in \mathcal{E}$ for all $x \in X$. The following 6 axioms for binary relations on $\mathcal{F}$ are necessary and sufficient for HEU:

Axiom 1: The binary relation $\succeq$ is complete and transitive. 
Axiom 2: If $f>g$, then $f \succ g$.

Axiom 3: $\quad y E \cap D x \sim y E \cap D^{\prime} x$ for all $x, y, E, D$ and $D^{\prime}$.

Axiom 4: If $y>x$ and $w>z$, then $y A x \succeq y B x$ implies $w A z \succeq w B z$.

Axiom 5: If $f, g \in \mathcal{F}^{e}$ and $f \succ g$, then there exists a partition $E_{1}, \ldots, E_{n}$ of $\Omega$ such that $l E_{i} f \succ m E_{i} g$ for all $i$.

Axiom 6: If $g \succeq f_{n} \succeq h$ for all $n$, then (i) $f_{n} \in \mathcal{F}^{e}$ converges pointwise to $f$ implies $g \succeq f \succeq h$ and (ii) $f_{n} \in \mathcal{F}$ converges uniformly to $f$ implies $g \succeq f \succeq h$.

Axioms 1, 2 and 4 are self-explanatory and standard. Axiom 3 says that all diffuse subsets of an ideal event are interchangeable. ${ }^{15}$ Axiom 5 is Savage's divisibility axiom applied to ideal events and guarantees the existence of a rich class of ideal events. Axiom 6 , our continuity axiom, ensures a continuous utility index and a countably additive prior.

The utility $W$ represents $\succeq$ if, for all $f, g \in \mathcal{F}, f \succeq g$ if and only if $W(f) \geq W(g)$.

Proposition 1: A binary relation satisfies Axioms $1-6$ if and only if there is an HEU that represents it.

An HEU has three parameters: the prior $\mu$ is the decision maker's perception of uncertainty, the utility index $v$ is her risk attitude for ideal acts, and $\alpha$ is her parameter of uncertainty aversion. Standard arguments ensure the usual uniqueness properties of these parameters: $\mu$ and $\alpha$ are unique and $v$ is unique up to positive affine transformations. For the remainder of this paper, we fix $\mu$, the agent's uncertainty perception, and let $W=(\alpha, v)$ denote an HEU.

Proposition 1 is closely related to Theorem 1 in Gul and Pesendorfer (2014). There we provide a representation theorem for expected uncertain utility theory of which HEU is a special case. Only Axiom 4 differs in the two representations. Axiom 4, above, is Savage's comparative probability axiom, P4. It requires that the betting preference is prize-independent. By contrast, expected uncertain utility theory assumes this axiom only for ideal events and permits prize-dependent betting preference for general events.

\footnotetext{
15 For a detailed discussion of this axiom, see Gul and Pesendorfer (2014).
} 


\section{Bets and Sources}

A bet on $A \subset \Omega$ is a binary act prize $y>x$ if $A$ occurs and prize $x$ if $A^{c}$ occurs. We write $y A x$ for a bet on $A$. If $W(y A x) \geq W(y B x)$ for some $y>x$, then, by Axiom 4 , the same inequality holds for all $x, y$ with $y>x$. Therefore, we say that $W$ prefers betting on $A$ to betting on $B$ and omit any reference to the two prizes. Henceforth, when we write $y A x$ it is understood that $y>x$. Let

$$
\mu_{*}(B)=\sup _{E \in \mathcal{E}_{\mu}, E \subset B} \mu(E)
$$

be the inner probability of an event $B$. Next, we express the utility of the bet $y A x$ in terms of inner probabilities. For this bet, the least favorable extension of $\mu$ assigns $A$ the (lower bound) probability $\mu_{*}(A)$ while the most favorable extension of $\mu$ assigns $A$ the (upper bound) probability $1-\mu_{*}\left(A^{c}\right) \cdot{ }^{16}$ Therefore,

$$
\begin{aligned}
W(y A x) & =\alpha\left[\mu_{*}(A) v(y)+\left(1-\mu_{*}(A)\right) v(x)\right] \\
& +(1-\alpha)\left[\left(1-\mu_{*}\left(A^{c}\right)\right) v(y)+\mu_{*}\left(A^{c}\right) v(x)\right]
\end{aligned}
$$

In general, the agent's uncertainty aversion, $\alpha$, affects his ranking of bets. For example, if $\mu_{*}(A)>\mu_{*}(B)$ and $\mu_{*}\left(A^{c}\right)>\mu_{*}\left(B^{c}\right)$, then $W(y A x)>W(y B x)$ if $W=(1, v)$ but the reverse inequality is true if $W=(0, v)$. Such reversals cannot occur when the two events belong to the same source.

Perhaps the best known example of multiple sources is the Ellsberg two-urn experiment. In it, subjects confront two urns: urn I consisting of balls with $n$ colors of unknown proportions and urn II with exactly one ball for each of the $n$ colors. In a single urn bet, the subjects are asked to bet on the color of a ball drawn from one of the urns. When comparing urn II bets, we would expect that a uniform probability over the $n$ colors represents the betting preference of any agent because the odds of drawing each color is $1 / n$. We would expect subjects to choose according to a uniform probability even among urn I bets. After all, the $n$ different color events are equally uncertain; that is, uncertainty

\footnotetext{
16 See, Lemma 2, in the appendix for a proof of these assertions.
} 
aversion provides no motive to favor one over another. Sources in our model share this feature: equally likely source events must be equally uncertain so that the agent's uncertainty attitude does not affect the ranking of bets.

The Ellsberg urn examples use a discrete state space whereas our model has a continuum of states. In our continuous setting, sources are (non-atomic) priors: $\pi \in \Pi$ is a source if, for every $(\alpha, v), A, B \in \mathcal{E}_{\pi}$,

$$
W(y A x) \geq W(y B x) \text { if and only if } \pi(A) \geq \pi(B)
$$

Moreover, if $A_{n} \in \mathcal{E}_{\pi}$ and $y A_{n} x$ converges pointwise to $y A x$, then

$$
W(y A x)=\lim W\left(y A_{n} x\right)
$$

We can compare our definition of a source to the Machina and Schmeidler (1992) definition of probabilistic sophistication. Machina and Schmeidler require that the domain of the prior be the set of all events and that the prior be relevant for all acts, not just bets. To see what the last statement means, let $G_{\pi}^{f}$ be the cumulative distribution of $f$ given the prior $\pi$. Then, Machina and Schmeidler (1992) would replace condition (5) above with (i) $W(f)=W(g)$ whenever $G_{\pi}^{f}=G_{\pi}^{g}$ and (ii) $W(f)>W(g)$ whenever $G_{\pi}^{f}$ stochastically dominates $G_{\pi}^{g}$.

Unlike Machina and Schmeidler, we allow the domain of the prior to be any $\sigma$-algebra and we restrict attention to bets. The first difference reflects our objective to study different sources and source preference; the second is for ease of exposition. It will turn out that the restriction to bets is inconsequential since, for $\mathcal{E}_{\pi}$-measurable acts, any prior that satisfies conditions (5) and (6) also satisfies the Machina-Schmeidler conditions (i) and (ii) above.

Proposition 2, below, demonstrates that HEU permits infinitely many distinct sources and that a power series characterizes each source. A function $\gamma:[0,1] \rightarrow[0,1]$ is a power series if there is a sequence $a=\left(a_{1}, a_{2}, \ldots\right)$ such that $a_{i} \in[0,1], \sum_{i=1}^{\infty} a_{i}=1$, and

$$
\gamma(t)=\sum_{i=1}^{\infty} a_{i} \cdot t^{i}
$$


Let $\Gamma$ be the set of all power series and let $\delta^{n}$ be the power series such that $\delta^{n}(t)=t^{n}$. For any prior $\pi \in \Pi$, we say that $\pi$ conforms to the power series $\gamma \in \Gamma$ if

$$
\mu_{*}(A)=\gamma(\pi(A))
$$

for all $A \in \mathcal{E}_{\pi}$.

Proposition 2: (i) A prior is a source if and only if it conforms to some power series; (ii) for every power series, there exists a source that conforms to it.

We write $\Sigma \subset \Pi$ for the collection of sources. For $\pi \in \Sigma$, we let $\gamma_{\pi}$ denote $\pi$ 's power series. Let $t=\pi(A)$ for $A \in \mathcal{E}_{\pi}$. To evaluate the utility of the bet $y A x$, we substitute (7) into expression (4) to obtain:

$$
\begin{aligned}
W(y A x) & =\alpha\left(\gamma_{\pi}(t) v(y)+\left(1-\gamma_{\pi}(t)\right) v(x)\right) \\
& +(1-\alpha)\left(\gamma_{\pi}(1-t) v(x)+\left(1-\gamma_{\pi}(1-t)\right) v(y)\right)
\end{aligned}
$$

Note that expression (8) depends only on the probability of the source event $(t=\pi(A))$ and is otherwise independent of $A$. As a result, if we replace $A$ with another, equally likely, source event $B$, then $W(y B x)=W(y A x)$ for all $\alpha$. Moreover, expression (8) is strictly increasing in $t$ and, therefore, all agents prefer bets on more likely events. Thus, the prior $\pi$ represents the betting preference for all $W=(\alpha, v)$. The continuity requirement follows from the continuity of $\gamma_{\pi}$.

For the proof of the only if part of part (i), note that the utility of a bet on a source event must be a function only of its probability; moreover, by the continuity requirement, this function must be strictly increasing and continuous. This implies that equation (8) must hold for some continuous, strictly increasing function from $[0,1]$ to $[0,1]$. The argument that this function must be a power series and that for each power series there is a conforming source is more involved. The main task in the proof of Proposition 2 is establishing these two facts. 


\section{Source Preference}

In this section, we define a comparative measure of uncertainty for sources and a comparative measure of uncertainty aversion. We relate the former to the source's power series and the latter to preference parameters $\alpha$ and $v$.

The utility $W$ is more uncertainty averse than the utility $\bar{W}=(\bar{\alpha}, \bar{v})$ if whenever $\bar{W}$ prefers betting on an event $E \in \mathcal{E}$ to betting on $A$, so does $W$.

Proposition 3: $\quad W=(\alpha, v)$ is more uncertainty averse than $\bar{W}=(\bar{\alpha}, \bar{v})$ if and only if $\alpha \geq \bar{\alpha}$.

We omit the formal proof of Proposition 3 because it is straightforward: the utility of a bet on an ideal event $E \in \mathcal{E}$ does not depend on $\alpha$ while, by equation (4), increasing $\alpha$ decreases the utility of a bet on a non-ideal event $A$. Thus, of the two decision makers, the one with a smaller $\alpha$ assigns relatively less value to non-ideal bets.

Next, we derive a notion of comparative source uncertainty from the above notion of comparative uncertainty aversion. Let $\pi^{o}, \pi \in \Sigma$ be two sources. We say that $W$ prefers source $\pi^{o}$ to source $\pi$ if

$$
\pi(A)=\pi^{o}(B) \text { implies } W(y B x) \geq W(y A x)
$$

whenever $A \in \mathcal{E}_{\pi}, B \in \mathcal{E}_{\pi^{o}}$. Source $\pi$ is more uncertain than source $\pi^{o}$ if there is $\bar{W}$ such that $W$ is more uncertainty averse than $\bar{W}$, implies $W$ prefers source $\pi^{o}$ to source $\pi$.

Proposition 4: $\quad$ Source $\pi$ is more uncertain than source $\pi^{o}$ if and only if $\gamma_{\pi}(t) \leq \gamma_{\pi^{o}}(t)$ for all $t$.

Proof: Let $\pi, \pi^{o} \in \Sigma$ be two sources and let $A \in \mathcal{E}_{\pi}, B \in \mathcal{E}_{\pi^{\circ}}$ be two events with the same source-probabilities $\left(\pi(A)=\pi^{o}(B)\right)$. Let $W=(\alpha, v)$ and let $\bar{W}=(\bar{\alpha}, \bar{v})$ be two utilities. Consider bets with prizes $y, x$ such that $y>x$ and normalize

$$
\begin{aligned}
& v(y)=\bar{v}(y)=1 \\
& v(x)=\bar{v}(x)=0
\end{aligned}
$$


By Proposition 3, if $W$ is more uncertainty averse than $\bar{W}$, then $0 \leq \bar{\alpha} \leq \alpha \leq 1$. Let $A \in$ $\mathcal{E}_{\pi}, B \in \mathcal{E}_{\pi^{o}}$ with $\pi(A)=t=\pi(B)$. Then, by expression (8) above, $W(y B x) \geq W(y A x)$ if and only if

$$
\alpha\left(\gamma_{\pi^{o}}(t)-\gamma_{\pi}(t)\right) \geq(1-\alpha)\left(\gamma_{\pi^{o}}(1-t)-\gamma_{\pi}(1-t)\right)
$$

If $\gamma_{\pi^{o}} \geq \gamma_{\pi}$ and if inequality (9) holds for $\alpha=\bar{\alpha}$, then the same inequality holds for all $\alpha \geq \bar{\alpha}$. It follows that $\pi$ is more uncertain than $\pi^{o}$. For the converse, note that if $\pi$ is more uncertain than $\pi^{o}$, then inequality (9) must hold for $\alpha=1$ and all $t$. It follows that $\gamma_{\pi^{o}} \geq \gamma_{\pi}$

The simplest kind of source preference is an unequivocal preference for less uncertain sources. The Ellsberg two-urn paradox provides empirical evidence for this ranking. Subjects are told that both urns contain 100 balls. Urn I contains red and green balls but no additional information about the composition of urn II is given while urn I has exactly 50 white and 50 black balls. Subjects are asked to rank $b, w, g, r$ where $c$ is a bet that delivers $y>0$ dollars if a ball of color $c \in\{b, w, r, g\}$ is drawn and zero otherwise. Subjects tend to be indifferent between $b$ and $w$ and between $r$ and $g$ but strictly prefer the first two bets to the last two. This is source preference in its purest form: decision makers prefer the less uncertain urn. ${ }^{17}$ Decision makers who exhibit this pattern are averse to uncertain sources: $W$ is averse to uncertain sources if $\gamma_{\pi^{\circ}} \leq \gamma_{\pi}$ implies that $W$ prefers source $\pi^{o}$ to source $\pi$.

Subsequent experimental research, however, has consistently yielded a more equivocal attitude towards more uncertain sources. Curley and Yates (1989) confirm aversion to uncertain sources when the odds of winning are large but document the reverse preference when the odds of winning are small. Heath and Tversky (1991) and Abdellaoui et al. (2011) identify a formally identical pattern: subjects prefer betting on the issue about which they are more knowledgeable when the (common) odds of winning are favorable but prefer betting on the issue about which they are less knowledgeable when the odds

\footnotetext{
17 With the aid of a procedure that Machina (2009) calls "orthogonal representation" even the Ellsberg single-urn paradox has been interpreted as relative uncertainty aversion. Machina (2009) credits Anscombe and Aumann (1963) with the idea of orthogonal representation.
} 
of winning are unfavorable. ${ }^{18}$ We call this pattern of behavior uncertainty loving at poor odds. The utility $W$ prefers source $\pi^{o}$ to source $\pi$ at odds $r$ if, for $y>x, A^{o} \in \mathcal{E}_{\pi^{o}}$, and $A \in \mathcal{E}_{\pi}$,

$$
\pi^{o}\left(A^{o}\right)=\pi(A)=r \text { implies } W\left(y A^{o} x\right) \geq W(y A x)
$$

The utility $W$ is uncertainty loving at poor odds if for any source $\pi \in \Sigma$, there is an uncertainty threshold $\bar{\gamma}$ and an odds-threshold $\bar{t}$ such that for $\pi^{o} \in \Sigma$ with $\gamma_{\pi^{o}} \leq \bar{\gamma}, W$ prefers $\pi^{o}$ to $\pi$ at odds $t \leq \bar{t}$.

Proposition 5: $W=(\alpha, v)$ is averse to uncertain sources if $\alpha=1$ and uncertainty loving at poor odds if $\alpha<1$.

To illustrate the second part of Proposition 5, we substitute $v(y)=1, v(x)=0$, and $\gamma_{\pi}=\delta^{n}$ into equation (8) above and obtain:

$$
W(y A x)=\alpha t^{n}+(1-\alpha)\left(1-(1-t)^{n}\right)
$$

Assume that $1>\alpha>1 / 2$ and let $n_{1}>n_{2}$. If $t \geq 1 / 2$, the agent prefers a bet on the source with power series $\delta^{n_{2}}$ to an equivalent bet on the more uncertain source with power series $\delta^{n_{1}}$. Thus, the agent prefers the bet on the less uncertain source if the odds of winning are good $(t \geq 1 / 2)$. By contrast, if the odds of winning are poor; that is, if $t<1-\alpha$, and if $n_{1}$ sufficiently large, the agent prefers a bet on the source with power series $\delta^{n_{1}}$ to an equivalent bet on a source with power series $\delta^{n_{2}}$; that is, she prefers the bet on the more uncertain source.

\subsection{Zhang Sources}

Zhang (2002) provides the following urn experiment: a single ball is drawn from an urn with two balls. The balls are either red, white, green or blue. It is known that exactly one ball is red or white and exactly one ball is green or blue. Therefore, the agent might plausibly perceive the events $A=\{$ red,white $\}$ and $B=\{$ green, blue $\}$ as equally likely. It is also known that exactly one ball is either red or green and exactly one ball is white or blue

\footnotetext{
18 Curley-Yates and Heath-Tversky differ in how they identify more or less uncertain environments. In Curley and Yates decision makers identify one source as subjectively more uncertain when its description is more "vague." In Heath and Tversky more uncertain environments are environments about which decision makers perceive themselves to be less knowledgeable.
} 
so that the agent plausibly perceives the events $C=\{$ red,green $\}$ and $D=\{$ white, blue $\}$ as equally likely. It is equally plausible that the agent would be indifferent between bets on any two of the four events $A, B, C$ and $D$. By contrast, an agent who exhibits the typical pattern in the Ellsberg paradox likely prefers betting on $A$ to betting on $F=\{r e d, b l u e\}$. Note that $F$ can be obtained as the union of intersections of events in $\{A, B, C, D\}$ since $F=(A \cap C) \cup(B \cap D)$. This suggests that the agent could plausibly perceive $A, B, C$ and $D$ to be part of a source that does not contain $F$. Therefore, the decision maker may have coherent betting preferences over collections of events and yet, it may not be possible to extend these preferences, in a coherent manner, to any algebra containing those events. Zhang's example describes a $\lambda$-system rather than a $\sigma$-algebra.

A class of events $\mathcal{A}$ is a $\lambda$-system if it contains $\Omega$ and is closed under the formation of complements and of countable disjoint unions. The pair $(\mathcal{A}, \pi)$ is a $\lambda$-prior if $\mathcal{A}$ is a $\lambda$-system and if $\pi$ is a countably additive, non-atomic probability measure on $\mathcal{A}$. The distinction between a weak source and a source, below, mirrors the distinction between a $\lambda$-prior and prior: the $\lambda$-prior $(\mathcal{A}, \pi)$ is a weak source if, for every $W=(\alpha, v), A, B \in \mathcal{A}$,

$$
W(y A x) \geq W(y B x) \text { if and only if } \pi(A) \geq \pi(B)
$$

Moreover, if $A_{n} \in \mathcal{E}_{\pi}$ and $y A_{n} x$ converges pointwise to $y A x$, then

$$
W(y A x)=\lim W\left(y A_{n} x\right)
$$

We call a weak source a Zhang-source if betting preferences on $(\mathcal{A}, \pi)$ cannot be extended to any $\sigma$-algebra that contains $\mathcal{A}$; specifically, we say that the weak source $(\mathcal{A}, \pi)$ is a Zhang-source if there is an event $F$ in the smallest $\sigma$-algebra that contains $\mathcal{A}$ such that for some $W=(\alpha, v)$ and all $A \in \mathcal{A}$ with $\pi(A)=1 / 2$ we have:

$$
\begin{aligned}
& W(y F x)=W\left(y F^{c} x\right) \\
& W(y A x)>W(y F x)
\end{aligned}
$$

The event $F$ matches the properties of the eponymous event in Zhang's example above: it is part of the smallest $\sigma$-algebra containing $\mathcal{A}$; the agent is indifferent between a bet on 
$F$ and a bet on $F^{c}$ and, finally, the agent prefers a bet on $A \in \mathcal{A}$ over a bet on $F$ if $A$ has probability $1 / 2$.

Proposition $2 \mathrm{w}(\mathrm{i})$ shows that a $\lambda$-prior $(\mathcal{A}, \pi)$ is a weak source if and only if it conforms to a power series $\gamma$, i.e., $\mu_{*}(A)=\gamma(\pi(A))$ for all $A \in \mathcal{A}$. Thus, weakening the definition of a source to $\lambda$-systems leaves our characterization result unaffected.

Proposition 2w: (i) $A \lambda$-prior is a weak source if and only if it conforms to a power series; (ii) there exist Zhang sources.

Part (ii) of Proposition 2w shows that our model can accommodate Zhang's example: some sources are defined on a $\lambda$-system and do not extend to a $\sigma$-algebra. ${ }^{19}$

\section{Sources and Lottery Utilities}

If an act is measurable with respect to the events of a source $\pi$, it is a random variable on the probability space $\left(\mathcal{E}_{\pi}, \pi\right)$. Therefore, we can map it to a lottery; that is, a cumulative over monetary prizes. In Proposition 5, below, we show that the utility of this act depends only on this implied lottery and so each source brings about a utility function over lotteries, a lottery utility. In this section, we characterize these lottery utilities and show how the uncertainty of the source (its power series) and the agent's uncertainty aversion (the parameter $\alpha$ ) affects their risk posture.

Recall that $X$ is the set of prizes. To simplify the presentation, we assume that $X$ contains 0 . Let $\mathcal{L}$ be the set of a cumulative distribution functions with support $X$. We write $F \gg G$ to denote stochastic dominance; that is, $F \leq G$ and $F \neq G$. We write $F_{n} \Rightarrow F$ to denote convergence in distribution; that is, $\lim _{n} F_{n}(x)=F(x)$ at every continuity point of $F$. The function $V: \mathcal{L} \rightarrow \mathbb{R}$ is a lottery utility if $V(F)>V(G)$ whenever $F \gg G$ and $\lim V\left(F_{n}\right)=V(F)$ whenever $F_{n} \Rightarrow F$. A lottery utility $V$ is risk averse if $V(F) \geq V(G)$ whenever $F$ is a mean preserving spread of $G$. The lottery utility $V$ is more risk averse than $\bar{V}$ if $\bar{V}(F) \geq \bar{V}(G)$ whenever $F$ is a compensated spread of $G$ for $V .{ }^{20}$

\footnotetext{
19 In the proof of Proposition 2w, we provide an example of a Zhang-source that conforms to a quadratic power series. The quadratic power series seems inessential to the construction and, therefore, we conjecture that a stronger result is true: for every power series $\gamma$, there is a Zhang source that conforms to $\gamma$.

20 The lottery $F$ is a compensated spread (Machina (1982)) of $G$ for the lottery utility $V$ if $V(F)=V(G)$ and if $F\left(x^{\prime}\right) \geq G\left(x^{\prime}\right)$ for $x \leq x^{\prime}$ and $F\left(x^{\prime}\right) \leq G\left(x^{\prime}\right)$ for $x \geq x^{\prime}$.
} 
For any source $\pi \in \Sigma$, let $\mathcal{F}_{\pi}$ denote the source-acts; that is, the collection of $\mathcal{E}_{\pi^{-}}$ measurable acts. The act $f \in \mathcal{F}_{\pi}$ yields the cumulative $G_{\pi}^{f} \in \mathcal{L}$ such that $G_{\pi}^{f}(x)=\pi(\{f \leq$ $x\})$. The lottery utility $V$ represents $W$ in source $\pi$ if for all $f, f^{\prime} \in \mathcal{F}_{\pi}$

$$
W(f) \geq W\left(f^{\prime}\right) \text { if and only if } V\left(G_{\pi}^{f}\right) \geq V\left(G_{\pi}^{f^{\prime}}\right)
$$

As we show in Proposition 6 below, the lottery utilities of HEU-agents are rank dependent expected utility (RDEU, Quiggin (1982)) in every source. RDEU is a well-known class of lottery utilities, commonly used in experimental studies that examine violations of the independence axiom (see, for example, the survey by Starmer (2000)). An RDEU has two parameters, a probability transformation function (PTF); that is, a continuous, increasing bijection $\tau:[0,1] \rightarrow[0,1]$, and a von Neumann-Morgenstern utility index. Let $T$ be the set of all PTFs. For $F \in \mathcal{L}$ and $\tau \in T$, the cumulative $F^{\tau}$ such that $F^{\tau}(x)=1-\tau(1-F(x))$ is the transformed lottery. The lottery utility $V$ is RDEU if and only if there is a $\tau \in T$ and a continuous, strictly increasing function $v: X \rightarrow \mathbb{R}$ such that the utility of the lottery $F$ is the expected utility of the transformed lottery $F^{\tau}$ :

$$
V(F)=\int v d F^{\tau}
$$

We write $V^{\tau v}$ for the RDEU with utility index $v$ and $\operatorname{PTF} \tau$.

The power series $\gamma \in \Gamma$ is an example of a PTF; for any $\gamma \in \Gamma$, the dual (or conjugate) $\hat{\gamma}$ of $\gamma$, defined as $\hat{\gamma}(t):=1-\gamma(1-t)$, is also a PTF and any weighted average of PTFs is a PTF. Proposition 6 characterizes the source utilities of HEU agents:

Proposition 6: The RDEU $V^{\tau v}$ such that $\tau=\alpha \gamma_{\pi}+(1-\alpha) \hat{\gamma}_{\pi}$ represents $W=(\alpha, v)$ in source $\pi$.

The utility index $v$ is the same for all sources but the PTF depends on the source and on $\alpha$. Note that each $\gamma$ is convex and, conversely, each $\hat{\gamma}$ is concave. Therefore, the PTF in each source is a weighted average of a convex PTF and its concave dual. For example, if $\gamma_{\pi}=\delta^{n}$ for some $n \geq 1$, then

$$
\begin{aligned}
\tau(t) & =\alpha \delta^{n}(t)+(1-\alpha) \hat{\delta}^{n}(t) \\
& =\alpha t^{n}+(1-\alpha)\left(1-(1-t)^{n}\right)
\end{aligned}
$$


RDEU utility exhibits first order risk aversion and the uncertainty aversion parameter $\alpha$ is related to the level of first order risk aversion. Fix a lottery $H \in \mathcal{L}$ with zero mean. For $x \in X$, define the lottery $H_{t x}$ as follows:

$$
H_{t x}(y)= \begin{cases}t H(y) & \text { for } y<x \\ t H(y)+1-t & \text { for } y \geq x\end{cases}
$$

Thus, $H_{t x}$ is a $t,(1-t)$ mixture of $H$ and the degenerate lottery that yields $x$ for sure. The risk premium of that lottery is $r(t)$, where $v(x-r(t))=V^{\tau v}\left(H_{t x}\right)$. If the utility index $v$ is differentiable, then first order risk aversion $\lim _{t \downarrow 0} \frac{r(t)}{t}$ is well defined and satisfies

$$
\lim _{t \downarrow 0} \frac{r(t)}{t}=-\left(\alpha E\left[H^{\gamma}\right]+(1-\alpha) E\left[H^{\hat{\gamma}}\right]\right)
$$

where $E[F]$ denotes the expectation of the lottery $F .{ }^{21}$ For $\gamma=\delta^{1}, V^{\tau v}$ is expected utility and first order risk aversion is zero. For $\gamma \neq \delta^{1}$, Jensen's inequality yields $E\left[H^{\gamma}\right]<0$ and $E\left[H^{\hat{\gamma}}\right]>0$ and, therefore, first order risk aversion increases in $\alpha$. This shows that more uncertainty averse agents (greater $\alpha$ ) exhibit greater first order risk aversion in every source. A greater $\alpha$ also implies greater risk aversion in general:

Proposition 7: If $\tau=\alpha \gamma+(1-\alpha) \hat{\gamma}$ and $\bar{\tau}=\bar{\alpha} \gamma+(1-\bar{\alpha}) \hat{\gamma}$, then $V^{v \tau}$ is more risk averse than $V^{\bar{v} \bar{\tau}}$ if and only if $v$ is a concave transformation of $\bar{v}$ and $\alpha \geq \bar{\alpha}$.

Abdellaoui, Baillon, Placido and Wakker (2012) estimate source-specific PTFs. Proposition 7 , above, shows that closely related source-utilities come about if the decision maker is an HEU maximizer. ${ }^{22}$ Their estimates reveal that typical sources have PTFs with an inverted S-shape. Kahnemann and Tversky (1979), Tversky and Kahneman (1992), Camerer and Ho, (1994), Wu and Gonzalez, (1996), Prelec (1998) and Abdellaoui (1998) provide earlier evidence in favor of PTFs with an inverted S-shape. More specifically, PTFs that provide a good fit are concave on $\left[0, t^{o}\right]$, convex on $\left[t^{o}, 1\right]$ for some $t^{o} \in(0,1 / 2)$ and cross the $45^{\circ}$ line between 0 and $1 / 2$. PTFs of the form

$$
\tau=\alpha \gamma+(1-\alpha) \hat{\gamma}
$$

\footnotetext{
21 For a derivation of first order risk aversion for RDEU see Segal and Spivak (1994), Proposition 4.

22 Abdellaoui et al. (2012) provide a model of source utilities but do not provide a corresponding model for general acts (acts not measurable with respect to a single source.) HEU does both - it provides a model for general acts and for acts measurable with respect to a single source. In this sense, we can interpret our model as a 'foundation' for their approach.
} 
have these properties whenever $1 / 2<\alpha<1$ and $\gamma$ places enough weight on higher order powers. More precisely, let $\gamma=\sum a_{i} \delta^{i}$ and $1 / 2<\alpha<1$. Then, the probability transformation function has the above mentioned properties if

$$
a_{1}+(1-\alpha) \sum_{i=2}^{\infty} i \cdot a_{i}>1
$$

For example, if $\gamma=a_{1} \delta^{1}+\left(1-a_{1}\right) \delta^{i}$, the PTF has the desired properties if $(1-\alpha) i>1$.

RDEUs with an inverted-S PTF are not globally risk averse because they prefer a gamble with sufficiently small odds of success over its expected value. As we show in Proposition 8, below, the only HEU utilities that are risk averse in every source are those with $\alpha=1$.

Proposition 8: For any $W=(\alpha, v)$, the following conditions are equivalent:

(i) $V^{\tau v}$ such that $\tau=\alpha \gamma+(1-\alpha) \hat{\gamma}$ is risk averse for every power series $\gamma$;

(ii) $W(g) \geq W(f)$ and $\beta \in[0,1]$ implies $W(\beta f+(1-\beta) g) \geq W(f)$;

(iii) $\alpha=1$ and $v$ is concave.

Proposition 8 relates risk aversion, as measured by the source utility $V^{\tau v}$, and uncertainty aversion as measured by the HEU parameter $\alpha$. Item (ii) in the above characterization is Schmeidler's notion of uncertainty aversion. Though formally identical, (ii) differs from Schmeidler's definition since acts yield monetary prizes in our model whereas they yield lotteries in Schmeidler's model. As a consequence, (ii) implies that the utility index $v$ must be concave while Schmeidler's definition has no such implication. ${ }^{23}$

In Gul and Pesendorfer (2014), we link uncertainty aversion to the agent's tendency to match the Ellsberg paradox. Here we have shown that the same parameter affects risk aversion in a systematic way: holding fixed the utility index $v$, more uncertainty averse agents exhibit greater first order risk aversion; they are more risk averse in any given source, and only maximally uncertainty averse agents can be risk averse in every source.

\footnotetext{
23 Schmeidler's definition implies that the capacity must be convex. HEU maximizers are Choquet expected utility maximizers (see Lemma 2 in the appendix) with a capacity that is convex if and only if $\alpha=1$.
} 


\section{Conclusion}

In this paper, we present a theory of subjective sources. Our model, Hurwicz expected utility, is a one-parameter extension of Savage's model that allows Ellsberg-paradox behavior. As in Savage, each agent has a prior and a utility index. The additional parameter, $\alpha$, measures the agent's uncertainty aversion. Despite the model's closeness to subjective expected utility theory, it admits a rich collection of subjective sources. Within each source, the agent maximizes rank dependent expected utility with a source-dependent probability transformation function.

An implication of our model is that risk postures vary substantially depending on how uncertain decision makers perceive a source to be. Thus, we would expect that measured risk aversion is context-dependent, just as the experimental and empirical literature on risk posture observes. Estimates of risk aversion measure two conceptually distinct features of a decision maker: risk and uncertainty attitudes (as measures by the parameter $\alpha$ and the utility index $v$ ) and how uncertain the decision maker perceives the particular source to be (as measured by its power series $\gamma$.) While the former is context independent, the latter is not. Confounding the two may lead to the incorrect conclusion that overall preferences are context-dependent.

\section{Appendix}

\subsection{Preliminaries}

\section{Cores, Diffuse Sets and Ideal Splits}

Let $\mu \in \Pi$ and let $\mathcal{E}_{\mu}^{+}=\left\{E \in \mathcal{E}_{\mu}: \mu(E)>0\right\}$. Recall that $\mu$ 's inner probability $\mu_{*}$ is defined as follows: $\mu_{*}(A)=\sup _{\substack{E \in \mathcal{E}_{\mu} \\ E \subset A}} \mu(E)$. We say that $A \subset \Omega$ and $B \subset \Omega$ are $\mu$-equivalent if $\mu_{*}(A \backslash B)=\mu_{*}(B \backslash A)=0$. Since $\mu$ is complete, $A$ and $B$ are $\mu$-equivalent if and only if $\mu(A \backslash B)=\mu(B \backslash A)=0$. Often, we write $A=B$ (or $A \subset B$ ) when in fact $A, B$ are only $\mu$-equivalent (or $\mu(B \backslash A)=0$ ). Since all of our measures are complete, this creates no problems.

For any $A \subset \Omega$, the core of $A$ is a set $E \subset A$ such that $\mu(E)=\mu_{*}(A)$. It is easy to verify that every $A$ has a core and that this core is unique up to $\mu$-equivalent sets. An 
alternative characterization of the core is as follows: $E$ is the core of $A$ if and only if every $E^{\prime} \in \mathcal{E}_{\mu}^{+}$such that $E^{\prime} \subset A$ intersects $E$.

For any nonempty, finite set $K$, let $K^{*}$ denote the set of all nonempty subsets of $K$. Let $\left\{A_{1}, \ldots, A_{n}\right\}$ be any collection of pairwise-disjoint subsets of $\Omega$ and $N=\{1, \ldots, n\}$. An ideal split is a collection of pairwise-disjoint, $\mu$-measurable sets $\left\{E^{J}\right\}_{J \in N^{*}}$ such that for all $J \in N^{*}$

$$
\begin{gathered}
\bigcup_{K \in J^{*}} E^{K} \subset \bigcup_{i \in J} A_{i} \\
\sum_{K \in J^{*}} \mu\left(E^{K}\right)=\mu_{*}\left(\bigcup_{i \in J} A_{i}\right)
\end{gathered}
$$

Thus, for any $J \in N^{*}, \bigcup_{K \in J^{*}} E^{K}$ is the core of $\bigcup_{i \in J} A_{i}$. We construct an ideal split for $\left\{A_{i}\right\}_{i \in N}$, inductively, as follows: when $J$ is a singleton, let $E^{J}$ be the core of $A_{i}$ for the unique $i \in J$. Then, for $J$ with cardinality $n+1$, let

$$
E^{J}=E \backslash\left(\bigcup_{\substack{K \in J^{*} \\ K \neq J}} E^{K}\right)
$$

where $E$ is the core of $\bigcup_{i \in J} A_{i}$.

Using the characterization of the core presented above, an inductive argument (on the cardinality of $K$ ) ensures that the construction above yields an ideal split. It is easy to verify that an ideal split is unique up to $\mu$-equivalent sets. Hence, we will say "the ideal split" rather than "an ideal split."

A set $D$ is diffuse if $\mu_{*}(D)=\mu_{*}\left(D^{c}\right)=0$. Let $\mathcal{D}$ be the set of all diffuse sets for $\mu$. In Gul and Pesendorfer (2014), we show that if the continuum hypothesis holds, there exists a collection of pairwise disjoint diffuse sets $\left\{D_{1}, D_{2}, \ldots\right\}$ such that $\bigcup_{i} D_{i}=\Omega$. If $D$ is a diffuse set, $A_{1}=D$ and $A_{2}=D^{c}$, then the ideal split of $\left\{A_{1}, A_{2}\right\}$ is $\left\{E^{\{1\}}, E^{\{2\}}, E^{\{1,2\}}\right\}$ such that $E^{\{1\}}=E^{\{2\}}=\emptyset$ and $E^{\{1,2\}}=\Omega$.

\section{Envelope Functions and the EUU Representation}

Let $\mathcal{F}^{*}$ be the set of all function from $\Omega$ to $X$. For any $f \in \mathcal{F}^{*}$, define $[f]_{1} \in \mathcal{E}_{\mu}$, the lower envelope of $f$ as follows: $f \geq[f]_{1}$ and $f \geq g$ implies $\mu\left(\left\{[f]_{1} \geq g\right\}\right)=1$. Thus, $[f]_{1}$ is the maximal $\mu$-measurable act that lies below $f$. In Gul and Pesendorfer (2014), we show 
that $[f]_{1}$ exists and is unique up to a set of measure 0 . The upper envelope $[f]_{2}$ of $f$ is defined analogously; i.e., by replacing $\geq$ in (i) and (ii) above with $\leq$.

Let $I=\{(x, y) \in X \times X \mid x \leq y\}$. A function $u: I \rightarrow \mathbb{R}$ is monotone if $x>x^{\prime}, y>y^{\prime}$ implies $u(x, y)>u\left(x^{\prime}, y^{\prime}\right)$. In Gul and Pesendorfer (2014), we replace Axiom 4 with the following:

Axiom $4^{*}:$ If $y>x$ and $w>z$, then $y E x \succeq y E^{\prime} x$ implies $w E z \succeq w E^{\prime} z$.

Hence, Axiom $4^{*}$ is weaker than Axiom 4 since the former applies only to ideal sets. Then, we prove that a binary relation $\succeq$ on $\mathcal{F}^{*}$ satisfies Axioms 1-3, $4^{*}, 5-6$ if and only if there is a prior $\mu$, a continuous, monotone $u: I \rightarrow \mathbb{R}$ such that the function $W^{*}$ below represents $\succeq$ :

$$
W^{*}(f)=\int u \circ\left([f]_{1},[f]_{2}\right) d \mu
$$

The proof of the representation theorem in Gul and Pesendorfer (2014) first shows that the representation holds for acts in $\mathcal{F}$; i.e., simple acts.

\subsection{Proof of Proposition 1}

We will first show that Axiom 4 ensures that there exists $\alpha \in[0,1]$ such that satisfies the following:

$$
u(x, y)=\alpha u(x, x)+(1-\alpha) u(y, y)
$$

Take any diffuse set $D$. Then, $[m D l]_{1}$ is the constant act $l$ and $[m D l]_{2}$ is the constant act $m$. It follows from equation $(*)$ above that $W(m D l)=u(l, m)$ and $W^{*}(m E l)=$ $\mu(E) u(m, m)+(1-\mu(E)) u(l, l)$. Define,

$$
\alpha=\frac{u(l, m)-u(l, l)}{u(m, m)-u(l, l)}
$$

Hence, $m E l \sim m D l$ if and only if $\mu(E)=\alpha$. Choose any such $E$ and $y>x$. Then, by Axiom 4, $y E x \sim y D x$ and since $W^{*}$ represents $\succeq$, we must have

$$
\alpha=\mu(E)=\frac{u(x, y)-u(x, x)}{u(y, y)-u(x, x)}
$$

as desired. 
Define, $v(x)=u(x, x)$ for all $x \in X$. Hence, we have shown that if $\succeq$ satisfies Axioms 1-6, there exists a prior $\mu, \alpha \in[0,1]$ and a utility index $v$ such that the function $W$ defined by

$$
W(f)=\alpha \int v \circ[f]_{1} d \mu+(1-\alpha) \int v \circ[f]_{2} d \mu
$$

represents $\succeq$.

For the remainder of this section, let $\left(x_{1}, \ldots, x_{n}\right)$ be the range of $f \in \mathcal{F}$ ordered so that $x_{1}<x_{2}<\ldots<x_{n}$ and let $A_{i}=f^{-1}\left(x_{i}\right)$.

Lemma 1: For all $f \in \mathcal{F}$ and $k=1,2$, (i) $\mu\left(\left\{[f]_{k} \subset f(\Omega)\right\}\right)=1$ and (ii) for all $i$, $E \subset[f]_{k}^{-1}\left(x_{i}\right)$ and $\mu(E)>0$ implies $E \cap A_{i} \neq \emptyset$.

Proof: We will prove Lemma 1 for $k=1$. The proof for $k=2$ is analogous and, therefore, omitted. It is enough for us to show that $\mu\left(\left\{[f]_{1} \subset\left(x_{i}, x_{i+1}\right)\right\}\right)=0$. If not, there exists $r$ such that $\mu\left(\left\{[f]_{1} \subset\left(x_{i}, x_{i+1}-1 / r\right)\right\}\right)>0$. Then, let $E=\left\{[f]_{1} \in\left(x_{i}, x_{i+1}-1 / r\right)\right\}$ and define $g=x_{i+1} E[f]_{1}$. Note that $\mu\left(\left\{[f]_{1}=g\right\}\right)<1$ and $f \geq g \geq[f]_{1}$, a contradiction. This concludes the proof of part (i). For part (ii), assume the contrary so that $E \subset[f]_{1}^{-1}\left(x_{i}\right)$, $\mu(E)>0$ and $E \cap A_{i}=\emptyset$ for some $E$ and $i$. By definition, $f \geq[f]_{1}$ and hence, by part (i), $f(\omega) \geq x_{i+1}$ for $\omega \in E$. But then, let $g=x_{i+1} E[f]_{1}$ and note again that $\mu\left(\left\{[f]_{1}=g\right\}\right)<1$ and $f \geq g \geq[f]_{1}$, a contradiction.

Lemma 2: $\quad$ For all $f \in \mathcal{F}$

$$
\begin{aligned}
W(f) & =\alpha\left(v\left(x_{1}\right)+\sum_{i=2}^{n}\left(v\left(x_{i}\right)-v\left(x_{i-1}\right)\right) \mu_{*}\left(\left\{f \geq x_{i}\right\}\right)\right) \\
& +(1-\alpha)\left(v\left(x_{1}\right)+\sum_{i=2}^{n}\left(v\left(x_{i}\right)-v\left(x_{i-1}\right)\right)\left(1-\mu_{*}\left(\left\{f \leq x_{i-1}\right\}\right)\right)\right.
\end{aligned}
$$

Proof: By (A1) it is sufficient to show that $\mu\left(\left\{[f]_{1} \geq x_{i}\right\}\right)=\mu_{*}\left(\left\{f \geq x_{i}\right\}\right)$ and $\mu\left(\left\{[f]_{2} \geq\right.\right.$ $\left.\left.x_{i}\right\}\right)=1-\mu_{*}\left(\left\{f \leq x_{i-1}\right\}\right)$ for all $i=2, \ldots, n$. Since the arguments for the two assertions are symmetric we will only proof the first. Since $f \geq[f]_{1}$ and $[f]_{1}$ is $\mathcal{E}_{\mu}$-measurable, it follows that $\mu_{*}\left(\left\{f_{1} \geq x\right\}\right) \geq \mu\left(\left\{[f]_{1} \geq x\right\}\right)$ for all $x \in X$. To prove the reverse inequality, assume $\mu_{*}\left(\left\{f \geq x_{i}\right\}\right)>\mu\left(\left\{[f]_{1} \geq x_{i}\right\}\right)$. This implies that there is $E \in \mathcal{E}_{\mu}$ such that 
$E \subset\left\{[f]_{1}<x_{i}\right\} \cap\left\{f \geq x_{i}\right\}$ with $\mu(E)>0$. By Lemma 1(i), $E \subset \bigcup_{j=1}^{i-1}\left\{[f]_{1}=x_{j}\right\}$. Then, Lemma 1(ii) implies $E \cap A_{j} \neq \emptyset$ for some $j<i$, contradicting the fact that $E \subset\left\{f \geq x_{i}\right\}$.

Proof of Proposition 1: For the only if part of the proof, we will show that

$$
\begin{aligned}
& \min _{\pi \in \Pi_{\mu}} \int v \circ f d \pi=\int v \circ[f]_{1} d \mu \\
& \max _{\pi \in \Pi_{\mu}} \int v \circ f d \pi=\int v \circ[f]_{2} d \mu
\end{aligned}
$$

We will prove the result for $\min _{\pi \in \Pi_{\mu}} \int v \circ f d \pi$. The proof for $\max _{\pi \in \Pi_{\mu}} \int v \circ f d \pi$ is analogous and, therefore, omitted.

Let $E_{i}=[f]_{1}^{-1}\left(x_{i}\right), J=\left\{i \mid E_{i} \neq \emptyset\right\}$ and let $B_{i j}=E_{i} \cap A_{j}$ for all $i, j \in J$. Then, let $J^{2}=\left\{(i, j) \in J \times J \mid B_{i j} \neq \emptyset\right\}$ and let $\mathcal{B}=\left\{B_{i j} \mid(i, j) \in J^{2}\right\}$. Note that since $J$ is finite, so is $\mathcal{B}$. Clearly, $\mathcal{B}$ is a partition of $\Omega$. Then, let $\mathcal{E}$ be the set of all sets that can be expressed as $\bigcup_{i j \in J^{2}} E_{i j} \cap B_{i j}$ for some collection of ideal sets $\left\{E_{i j}\right\}_{i j \in J^{2}}$ such that $E_{i j} \subset E_{i}$ for all $i, j$. It is easy to verify that $\mathcal{E}$ is the is the smallest $\sigma$-algebra that contains both $\mathcal{E}_{\mu}$ and $\left\{A_{1}, \ldots, A_{n}\right\}$. Note also that if

$$
\bigcup_{i j \in J^{2}} E_{i j} \cap B_{i j}=\bigcup_{i j \in J^{2}} \hat{E}_{i j} \cap B_{i j}
$$

for $\bigcup_{i j \in J^{2}} E_{i j} \cap B_{i j}$ and $\bigcup_{i j \in J^{2}} \hat{E}_{i j} \cap B_{i j}$ in $\mathcal{E}$, then $E_{i i}$ and $\hat{E}_{i i}$ are $\mu$-equivalent for all $i \in J$. To see this, suppose $\mu\left(E_{i i} \backslash \hat{E}_{i i}\right)>0$ for some $i i \in J^{2}$. Let $E=E_{i i} \backslash \hat{E}_{i i}$. Then, by Lemma 1(ii), $E \cap A_{i} \neq \emptyset$. But, $E \cap A_{i} \subset E_{i i} \cap B_{i i}$ and $\left(E \cap A_{i}\right) \cap\left(\bigcup_{j \in J} \hat{E}_{i j} \cap B_{i j}\right)=\emptyset$, contradicting $(* *)$ above.

Define, $\pi$ as follows:

$$
\pi\left(\bigcup_{i j \in J^{2}} E_{i j} \cap B_{i j}\right)=\sum_{i \in J} \mu\left(E_{i i}\right)
$$

Note that $\pi$ is the unique extension of $\mu$ to $\mathcal{E}$ such that $\pi\left(E_{i} \cap A_{i}\right)=\mu\left(E_{i}\right)$ for all $i \in J$. That $\pi$ is a prior follows from the fact that $\mu$ is a prior. Note also that $\pi\left(A_{i}\right)=\pi\left(E_{i} \cap A_{i}\right)=\mu\left(E_{i}\right)$ and hence

$$
\int v \circ[f]_{1} d \mu=\sum_{i} v\left(x_{i}\right) \mu\left(E_{i}\right)=\sum_{i} v\left(x_{i}\right) \pi\left(E_{i}\right)=\int v \circ f d \pi
$$


Then, to conclude the proof, it is sufficient for us to show that for any $\hat{\pi}$ such that $\left\{A_{1}, \ldots, A_{n}\right\} \subset \mathcal{E}_{\hat{\pi}}, \mathcal{E}_{\mu} \subset \mathcal{E}_{\hat{\pi}}$ and $\hat{\pi}(E)=\mu(E)$ for all $E \in \mathcal{E}$, we must have $\int v \circ f d \hat{\pi} \geq$ $\int v \circ[f]_{1} d \mu$. But this obvious since $x_{i}=\min _{\omega \in E_{i}} f(\omega)$.

\subsection{Proof of Propositions 2 and $2 \mathrm{w}$}

\subsubsection{Proof of Proposition 2(i) and 2w(i):}

Since every prior is a $\lambda$-prior and every weak-source on a $\sigma$-algebra is a source, $2 \mathrm{w}(\mathrm{i})$ implies 2(i) and therefore, we will only prove the former.

Claim: Let $x_{n i}$ and $y_{n i}$ be two arrays such that $0 \leq x_{n i}, y_{n i} \leq 1$ for all $n, i=1,2, \ldots$ If, for some $t, \gamma \in(0,1)$ and sequence $z_{i} \in[0,1]$,

(1) $\sum_{i} x_{n i}=1$ and $\sum_{i} x_{n i} \cdot y_{n i}=\gamma$ for all $n$,

(2) $y_{n i} \leq t^{i}$ for all $n, i, \lim _{n \rightarrow \infty} y_{n i}=t^{i}$, and $\lim _{n \rightarrow \infty} x_{n i}=z_{i}$ for all $i$, then,

$$
\sum_{i} z_{i} \cdot t^{i}=\gamma
$$

Proof of the Claim: For any $\epsilon>0$, choose $k$ such that $\sum_{i=k+1}^{\infty} t^{i}<\epsilon$. Then,

$$
\sum_{i=1}^{k} x_{n i} \cdot y_{n i}=\gamma-\sum_{i=k+1}^{\infty} x_{n i} \cdot y_{n i} \geq \gamma-\left(\sum_{i=k+1}^{\infty} x_{n i}\right) \cdot\left(\sum_{i=k+1}^{\infty} y_{n i}\right) \geq \gamma-\epsilon
$$

and hence $\gamma-\epsilon \leq \sum_{i=1}^{k} x_{n i} \cdot y_{n i} \leq \gamma$. Then, for any $K>k$, letting $n$ go to infinity yields

$$
\gamma-\epsilon \leq \sum_{i=1}^{K} z_{i} \cdot t^{i} \leq \gamma
$$

Thus, we have $\sum_{i=1}^{\infty} z_{i} \cdot t^{i}=\gamma$ as desired.

Let $W=(\mu, \alpha, v), \alpha=1$ and let $(\mathcal{A}, \pi)$ be a weak source. Then, $W(y A x)=$ $v(y) \mu_{*}(A)+v(x)\left(1-\mu_{*}(A)\right)$. Since, $W(y A x) \geq W(y B x)$ if and only if $W(y A x) \geq W(y B x)$ whenever $A, B \in \mathcal{A}, y>x$ and $y^{\prime}>x^{\prime}$, we conclude that $\pi(A) \geq \pi(B)$ if and only if $\mu_{*}(A) \geq \mu_{*}(B)$. Setting $\gamma(t)=\mu_{*}(A)$ for some $A$ such that $\pi(A)=t$ ensures that $\gamma:[0,1] \rightarrow[0,1]$ is well-defined. 
Next, we show that $\gamma$ is a power series. Let $N=\left\{1, \ldots, 2^{n}\right\}, \mathcal{B}_{n}=\left\{A_{j}\right\}_{j \in N} \subset \mathcal{A}$ be a partition of $\Omega$ such that and $\pi\left(A_{j}\right)=1 / 2^{n}$ for all $j$. Recall that $N^{*}$ is the set of all nonempty subsets of any $\operatorname{set} N$. In section 7.1 , we noted that

$$
\mu\left(E^{J}\right)=\mu_{*}\left(\bigcup_{j \in J} A_{i}\right)-\sum_{\substack{L \in J^{*} \\ L \neq J}} \mu\left(E^{L}\right)
$$

for all $J \in N^{*}$ and $\left\{E^{K}\right\}_{K \in N^{*}}$ is the ideal split of $\mathcal{B}_{n}$. Since $\pi\left(\bigcup_{j \in J} A_{j}\right)=\pi\left(\bigcup_{j \in L} A_{j}\right)$ whenever $|L|=|J|$, a straightforward inductive argument reveals that $\mu\left(E^{J}\right)=\mu\left(E^{L}\right)$ whenever $|J|=|L|$. Hence, we can define

$$
a_{n i}=\left(\begin{array}{c}
2^{n} \\
i
\end{array}\right) \mu\left(E^{J}\right)
$$

for $J$ such that $|J|=i$. For $i>2^{n}$, set $a_{n i}=0$. Since $\left\{E^{J}\right\}$ is a partition of $\Omega$,

$$
\sum_{i=1}^{\infty} a_{n i}=\sum_{i=1}^{2^{n}} a_{n i}=1
$$

for all $n$.

Hence, $0 \leq a_{n i} \leq 1$ for all $n, i$ and therefore, with a diagonal argument, we can find a subsequence $n_{m}$ and a sequence $a_{i}, i=1, \ldots$ such that $\lim _{m \rightarrow} a_{n_{m} i}=a_{i}$ for all $i$. Without loss of generality, we will assume that this subsequence is the sequence $n$ itself.

Let $t \in(0,1)$ be any dyadic number; hence $t=k / 2^{m}$ for integers $k, m>0$. Then, for $n \geq m$ set $K_{n}=\left\{1, \ldots, k 2^{n-m}\right\}$ and note that equation (A2) implies,

$$
\begin{aligned}
\gamma(t)=\mu_{*}\left(\bigcup_{i \in K_{n}} A_{i}\right) & =\sum_{L \in K_{n}^{*}} \mu\left(E^{L}\right) \\
& =\sum_{i=1}^{k 2^{n-m}} \sum_{\substack{L \in K_{n} \\
|L|=i}} a_{n i} \cdot\left(\begin{array}{c}
2^{n} \\
i
\end{array}\right)^{-1} . \\
& =\sum_{i=1}^{k 2^{n-m}} a_{n i} \cdot\left(\begin{array}{c}
k 2^{n-m} \\
i
\end{array}\right)\left(\begin{array}{c}
2^{n} \\
i
\end{array}\right)^{-1}
\end{aligned}
$$

Note that $\lim _{n \rightarrow \infty}\left(\begin{array}{c}k 2^{n-m} \\ i\end{array}\right)\left(\begin{array}{c}2^{n} \\ i\end{array}\right)^{-1}=\left(k / 2^{m}\right)^{i}=t^{i}$. To see this, observe that $\left(\begin{array}{c}k 2^{n-m} \\ i\end{array}\right)\left(\begin{array}{c}2^{n} \\ i\end{array}\right)^{-1}$ is the probability of drawing $i$ red balls in $i$ tries, without replacement, from an urn that 
has $2^{n}$ balls, $k 2^{n-m}$ of which are red, while $t^{i}$ is the corresponding probability when the draws are made with replacement. As $n$ goes to infinity, the two probabilities become the same.

Set $x_{n i}=a_{n i}, y_{n i}=\left(\begin{array}{c}k 2^{n-m} \\ i\end{array}\right)\left(\begin{array}{c}2^{n} \\ i\end{array}\right)$ for all $n$ and $i \leq 2^{n-m}$. For $i>2^{n-m}$, set $x_{n i}=y_{n i}=0$. Then, set $z_{i}=a_{i}$ for all $i$ and $\gamma=\gamma(t)$ and verify that the assumptions of the claim are met to conclude

$$
\gamma(t)=\sum_{i=1}^{\infty} a_{i} \cdot t^{i}
$$

for every dyadic $t \in(0,1)$. Then, let $\hat{\gamma}(t)=\sum_{i=1}^{\infty} a_{i} \cdot t^{i}$ for all $t \in[0,1]$. Both $\hat{\gamma}$ and $\gamma$ are continuous and agree on a dense subset of $[0,1]$. It follows that $\hat{\gamma}=\gamma$ and $\sum_{i=1}^{\infty} a_{i}=1$ as desired.

To prove the converse, suppose for some $\lambda$-prior $(\mathcal{A}, \pi)$ and power series $\gamma, \mu_{*}(A)=$ $\gamma(\pi(A))$ for all $A \in \mathcal{A}$. Then, substituting $\pi(A)$ into expression (8), we get

$$
W(y A x)=v(x)+\gamma(\pi(A))[v(y)-v(x)]+[1-\gamma(1-\pi(A))](1-\alpha)(v(y)-v(x))
$$

Since this expression is continuous and strictly increasing in $\pi(A)$ for every $(\alpha, v)$ and $y>x$, the conditions for a weak source are satisfied.

\subsubsection{Proof of Proposition 2(ii):}

Fix the prior $\mu$ and the power series $\gamma$ such that $\gamma(t)=\sum a_{i} \cdot t^{i}$ for $a \in \mathcal{Z}$. We will construct a prior, $\left(\mathcal{E}_{\pi}, \pi\right)$, such that $\mu_{*}(A)=\gamma(\pi(A))$ for all $A \in \mathcal{E}_{\pi}$. Since $\mu$ is convex valued, for every cumulative $F$, there is $g \in \mathcal{F}_{\mu}^{*}$ such that $G_{\mu}^{g}=F$. Choose a countable set $Y=\left\{z_{n}\right\} \subset X$ such that $z_{i}<z_{i+1}$ for all $i$ and let $g_{0} \in \mathcal{F}_{\mu}$ be such that $\mu\left(g_{0}^{-1}\left(z_{i}\right)\right)=a_{i}$. As we noted in section 7.1, there exists a collection of pairwise disjoint diffuse sets $\left\{D_{1}, D_{2}, \ldots\right\}$ such that $\bigcup_{i} D_{i}=\Omega$. For $i=1,2, \ldots$, choose $g_{i} \in \mathcal{F}_{\mu}^{*}$ such that (1) $g_{i}$ is uniformly distributed on $[l, m]$ and $(2) g_{0}, g_{1}, \ldots$ is a sequence of independent random variables. Define $g \in \mathcal{F}^{*}$ as follows:

$$
g(\omega)= \begin{cases}g_{i}(\omega) & \text { if } \omega \in D_{i} \cap g_{0}^{-1}\left(z_{j}\right) \text { for } i<j \\ g_{j}(\omega) & \text { if } \omega \in \bigcup_{i \geq j} D_{i} \cap g_{0}^{-1}\left(z_{j}\right)\end{cases}
$$

Let $\mathcal{E}^{g}$ be the $\sigma$-algebra generated by $g$ and let $\nu$ be the unique measure on the Borel sets of $X$ such that $\nu[l, x]=\frac{x-l}{m-l}$ for all $x \in X$. Then, define the probability measure 
$\left(\mathcal{E}^{g}, \pi^{g}\right)$ as follows: $\pi^{g}\left(g^{-1}(B)\right)=\nu(B)$ for every Borel $B \subset X$. Finally, let $\pi$ be the completion $^{24}$ of $\pi^{g}$. We claim that $\pi$ is a prior and that

$$
\mu_{*}(A)=\gamma(\pi(A))
$$

Since $\nu$ is nonatomic, so are $\pi$ and $\pi^{g}$. Hence, $\pi$ is a prior.

Next, we prove (A4) for $A \in \mathcal{E}^{g}$. Let $A=g^{-1}(B)$ and let $E_{i}^{A}=g_{0}^{-1}\left(z_{i}\right) \cap \bigcap_{j=1}^{i} g_{j}^{-1}(B)$. Clearly, $E_{i}^{A} \in \mathcal{E}_{\mu}$ and $E_{i}^{A} \subset A$ and therefore, $\bigcup_{i \geq 1} E_{i}^{A} \subset A$. Also, the sets $E_{i}^{A}$ are pairwise disjoint and therefore,

$$
\mu\left(\bigcup_{i \geq 1} E_{i}^{A}\right)=\sum_{i \geq 1} \mu\left(E_{i}^{A}\right)=\sum_{i \geq 1} a_{i} \cdot(\nu(B))^{i}=\gamma(\pi(A))
$$

Hence, $\mu_{*}(A) \geq \gamma(\pi(A))$. Suppose $E \in \mathcal{E}_{\mu}, \mu(E)>0$ and $E \not \subset \bigcup_{i \geq 1} E_{i}^{A}$. Let $E^{\prime}=$ $E \backslash\left(\bigcup_{i \geq 1} E_{i}^{A}\right)$ and let $i$ be such that $E^{\prime \prime}:=E^{\prime} \cap g_{0}^{-1}\left(z_{i}\right) \neq \emptyset$. It follows that $E^{\prime \prime} \subset$ $g_{0}^{-1}\left(z_{i}\right) \cap\left(E_{i}^{A}\right)^{c}$. This, in turn, implies that $E^{\prime \prime} \cap g_{j}^{-1}\left(B^{c}\right) \cap D_{j} \neq \emptyset$ for some $j \leq i$ and, therefore, $E \not \subset g^{-1}(B)$. We conclude that $\mu_{*}(A) \leq \mu\left(\bigcup_{i \geq 1} E_{i}^{A}\right)=\gamma(\pi(A))$ as desired.

It is easy to verify that for any $A \in \mathcal{E}_{\pi}$, there is $A_{1} \subset A \subset A_{2}$ such that $A_{1}, A_{2} \in \mathcal{E}^{g}$ and $\pi^{g}\left(A_{1}\right)=\pi^{g}\left(A_{2}\right)$. Therefore, since (A4) holds for all $A \in \mathcal{E}^{g}$, it also holds for all $A \in \mathcal{E}_{\pi}$. Hence, $\pi$ conforms to the power series $\gamma$ and part (i) of this proposition ensures that $\pi$ is a source.

\subsubsection{Proof of Proposition 2w(ii)}

Let $D_{1}, D_{2}$ be a partition of $\Omega$ into two diffuse sets. Choose four independent, uniformly distributed random variables, $g_{i}, i=1,2,3,4$ on $\left(\mathcal{E}_{\mu}, \mu\right)$ with support $X$. Let

$$
f_{1}(\omega)= \begin{cases}g_{1}(\omega) & \text { if } \omega \in D_{1} \\ g_{2}(\omega) & \text { if } \omega \in D_{2}\end{cases}
$$

and let

$$
f_{2}(\omega)= \begin{cases}g_{3}(\omega) & \text { if } \omega \in D_{1} \\ g_{4}(\omega) & \text { if } \omega \in D_{2}\end{cases}
$$

24 The completion, $(\hat{\mathcal{E}}, \hat{\pi})$, of a probability measure, $\left(\mathcal{E}_{o}, \pi_{o}\right)$, exists and is complete. Moreover, $\mathcal{E}_{o} \subset \hat{\mathcal{E}}$ and for all $B \in \hat{\mathcal{E}}$, there are $A, C \in \mathcal{E}_{o}$ such that $A \subset B \subset C$ and $\pi_{o}(A)=\hat{\pi}(B)=\pi_{o}(C)$. See, Billingsley (1995), page 49 . 
Let $\nu$ be the unique measure on the Borel sets of $X$ such that $\nu[l, x]=\frac{x-l}{m-l}$ for all $x \in X$. For $i=1,2$, let $\left(\mathcal{E}_{\pi_{i}}, \pi_{i}\right)$ be the source constructed from $f_{i}$ as described in the proof of Proposition 2(ii). It is easy to verify that the power series of both of these sources is $\delta^{2}$. To simplify the notation below, we write $\mathcal{E}_{1}$ for $\mathcal{E}_{\pi_{1}}$ and $\mathcal{E}_{2}$ for $\mathcal{E}_{\pi_{2}}$.

Let $\mathcal{E}_{1}^{\prime}=\left\{(B \cup C) \cap F \mid B \in \mathcal{E}_{1}, C, F \in \mathcal{E}_{2}, \pi_{2}(C)=0, \pi_{2}(F)=1\right\}$. For $A \in \mathcal{E}_{1}^{\prime}$, call $(B \cup C) \cap F$ a $\pi_{1}^{\prime}$-breakdown of $A$ if $A=(B \cup C) \cap F, B \in \mathcal{E}_{1}, C, F \in \mathcal{E}_{2}, \pi_{2}(C)=0$ and $\pi_{2}(F)=1$. We claim that $\mathcal{E}_{1}^{\prime}$ is a $\sigma$-algebra. To see this, first note that $\Omega=(\Omega \cup \emptyset) \cap \Omega \in \mathcal{E}_{1}^{\prime}$. Then, Let $(B \cup C) \cap F$ be a $\pi_{1}^{\prime}$-breakdown of $A$. Then, $A^{c}=\left(B^{c} \cup F^{c}\right) \cap\left(C^{c} \cup F^{c}\right)$. Since $\pi_{2}(C)=0$ and $\pi_{2}(F)=1$, we have $\pi_{2}\left(C^{c}\right)=1$ and $\pi_{2}\left(F^{c}\right)=0 ;$ since $\pi_{2}$ is complete and $\pi_{2}\left(C^{c}\right)=1$, we have $\left(C^{c} \cup F^{c}\right) \in \mathcal{E}_{2}$ and $\pi_{2}\left(C^{c} \cup F^{c}\right)=1$. Therefore, $\left(B^{c} \cup F^{c}\right) \cap\left(C^{c} \cup F^{c}\right)$ is a $\pi_{1}^{\prime}$-breakdown of $A^{c}$ and hence $A^{c} \in \mathcal{E}_{1}^{\prime}$.

To conclude the proof that $\mathcal{E}_{1}^{\prime}$ is a $\sigma$-algebra, we will prove that countable unions of elements of $\mathcal{E}_{1}^{\prime}$ are in $\mathcal{E}_{1}^{\prime}$. Let $\left(B^{i} \cup C^{i}\right) \cap F^{i}$ be a $\pi_{1}^{\prime}$-breakdown of $A^{i}$ and let $A=\bigcup_{i=1}^{\infty} A^{i}$. Then, let $B=\bigcup_{i} B^{i}, C=\bigcup_{i} C^{i}, G=\bigcap_{i} F^{i}$ and $H=\bigcup_{i} F^{i}$. Clearly, $B \in \mathcal{E}_{1}$ and $C, G, H \in \mathcal{E}_{2}, \pi_{2}(G)=\pi_{2}(H)=1$ and $(B \cup C) \cap G \subset A \subset(B \cup C) \cap H$. Hence, there exists $F$ satisfying $G \subset F \subset H$ such that $A=(B \cup C) \cap F$. Since $\pi_{2}$ is complete, $F \in \mathcal{E}_{2}$ and $(B \cup C) \cap F$ is a $\pi_{1}^{\prime}$-breakdown of $A$. Hence, $A \in \mathcal{E}_{1}^{\prime}$.

Extend $\pi_{1}$ to $\mathcal{E}_{1}^{\prime}$ by letting $\pi_{1}(A)=\pi_{1}(B)$ given any $\pi_{1}^{\prime}$-breakdown $(B \cup C) \cap F$ of $A$. Let $\pi_{1}^{\prime}$ denote this extension. To prove that $\pi_{1}^{\prime}$ is well-defined, we make the following observation: given any $\pi_{1}^{\prime}$-breakdown, $(B \cup C) \cap F$ of $A$,

$$
\mu_{*}(B \cap F)=\mu_{*}(B)=\mu_{*}(B \cup C)=\mu_{*}(A)
$$

To see why this is the case, note that $\pi_{2}(F)=1$ implies $\mu_{*}(F)=1$ and since $\mu$ is complete, $F \in \mathcal{E}_{\mu}$ and $\mu(F)=1$. It follows that $\mu_{*}(B \cap F)=\mu_{*}(B)$. Since $\pi_{2}(C)=0$, we have $\pi_{2}\left(C^{c}\right)=1$ and therefore, $\mu_{*}\left(C^{c}\right)=\mu\left(C^{c}\right)=1$. Hence, $C \in \mathcal{E}_{\mu}$ and $\mu(C)=0$. Therefore, $\mu_{*}(B)=\mu_{*}(B \cup C)$. Since $B \cap F \subset A \subset B \cup C$, the last equality implies $\mu_{*}(A)=\mu_{*}(B \cup C)$.

To verify that $\pi_{1}^{\prime}$ is well-defined, let $(B \cup C) \cap F=(\hat{B} \cup \hat{C}) \cap \hat{F}$ be two $\pi_{1}^{\prime}$-breakdowns of $A \in \mathcal{E}_{1}^{\prime}$. It follows from equation (A5) that $\mu_{*}(B)=\mu_{*}(\hat{B})$.

Next, we will show that $\pi_{1}^{\prime}$ is countably additive. Let $A^{i} \in \mathcal{E}_{1}^{\prime}$ be a pairwise disjoint sequence. Then, let $\left(B^{i} \cup C^{i}\right) \cap F^{i}$ be a $\pi_{1}^{\prime}$-breakdown of $A^{i}$ and let $A=\bigcup_{i=1}^{\infty} A^{i}$. Define, 
$B=\bigcup_{i} B^{i}$ and $C=\bigcup_{i} C^{i}$. We established above (while proving that $\mathcal{E}_{1}^{\prime}$ is closed under countable unions) that there is $F \in \mathcal{E}_{2}$ such that $\pi_{2}(F)=1$ and $(B \cup C) \cap F$ is a $\pi_{1}^{\prime}$ breakdown of $A^{i}$. Then, $\pi_{1}^{\prime}(A)=\pi_{1}(B)=\sum_{i} \pi_{1}\left(B^{i}\right)=\sum_{i} \pi_{1}^{\prime}\left(A^{i}\right)$ as desired.

Since $\pi_{1}$ is a source, it is nonatomic. Then, $\pi_{1}^{\prime}$ is also nonatomic. To establish that $\pi_{1}^{\prime}$ is a prior, all that remains is to show that it is complete: let $A \subset(B \cup C) \cap F$ for some $B$ such that $\pi_{1}(B)=0$. Then, $A=((A \cap B) \cup(A \cap C)) \cap F$. Since $\pi_{1}, \pi_{2}$ are complete, we have $(A \cap B) \in \mathcal{E}_{1}$ and $A \cap C \in \mathcal{E}_{2}$ and therefore, $A \in \mathcal{E}_{1}^{\prime}$ proving that $\pi_{1}^{\prime}$ is complete.

Equation (A5) together with the argument made in the last part of the proof of Proposition 2(ii) establishes that $\mu_{*}(A)=\left(\pi_{1}^{\prime}(A)\right)^{2}$ for all $A \in \mathcal{E}_{1}^{\prime}$. Then, Proposition 2 ensures that $\left(\mathcal{E}_{1}^{\prime}, \pi_{1}^{\prime}\right)$ is a source with power series $\delta^{2}$. Define $\mathcal{E}_{2}^{\prime}, \pi_{2}^{\prime}$ and $\pi_{2}^{\prime}$-breakdown analogously by reversing the roles of $\mathcal{E}_{1}, \pi_{1}$ and $\mathcal{E}_{2}, \pi_{2}$. We claim that (1) $\left[A_{i} \in \mathcal{E}_{i}\right.$ for $\left.i=1,2, \pi_{1}^{\prime}\left(A_{1}\right) \cdot \pi_{2}^{\prime}\left(A_{2}\right)>0\right]$ implies $A_{1} \cap A_{2} \neq \emptyset$ and $(2) A \in \mathcal{E}_{i}^{\prime}, \pi_{i}^{\prime}(A)=0$ for some $i=1,2$ implies $\left[A \in \mathcal{E}_{1}^{\prime} \cap \mathcal{E}_{2}^{\prime}\right.$ and $\left.\pi_{1}^{\prime}(A)=\pi_{2}^{\prime}(A)=0\right]$.

Let $\left(B_{i} \cup C_{i}\right) \cap F_{i}$ be a $\pi_{i}^{\prime}$-breakdown of $A_{i}$. Assertion (1) follows easily from the way $\mathcal{E}_{1}^{\prime}, \mathcal{E}_{2}^{\prime}$ are constructed. In particular, it follows from the fact that the functions $g_{1}, g_{2}, g_{3}, g_{4}$ are independent. To prove (2), without loss of generality, let $i=1$ and $(B \cup C) \cap F$ be a $\pi_{1}^{\prime}$-breakdown. If $\pi_{1}^{\prime}(B)=0$ then, since $\pi_{1}$ is complete, $B \cap F \in \mathcal{E}_{1}^{\prime}$. Similarly, since $\pi_{2}$ is complete, $\pi_{2}(C \cap F)=0$. Then, $[(C \cap F) \cup(B \cap F)] \cap \Omega$ is a $\pi_{2}^{\prime}$-breakdown of $A$ and $\pi_{2}^{\prime}(A)=\pi_{2}(C \cap F)=0$.

Let $\mathcal{A}=\mathcal{E}_{1}^{\prime} \cup \mathcal{E}_{2}^{\prime}$ and let $\pi(A)=\pi_{i}(A)$ if $A \in \mathcal{E}_{i}^{\prime}$. First, we will show that $\pi$ is welldefined; that is, if $A \in \mathcal{E}_{1}^{\prime} \cap \mathcal{E}_{2}^{\prime}$, then $\pi_{1}^{\prime}(A)=\pi_{2}^{\prime}(A)$. If $\pi_{1}^{\prime}(A)=0,(2)$ above establishes the desired result; if $\pi_{1}^{\prime}(A)>0$, then (2) implies $\pi_{2}^{\prime}(A)>0$. So, either $\pi_{1}^{\prime}(A) \cdot \pi_{2}^{\prime}(A)=1$ and there is nothing to prove or $\pi_{i}^{\prime}(A)<1$ for some $i \in\{1,2\}$. Then, (1) yields $A^{c} \cap A \neq \emptyset$, a contradiction.

We will show that $\mathcal{A}$ is a $\lambda$-system and that $\pi$ is a $\lambda$-prior. Since $\mathcal{E}_{1}^{\prime}, \mathcal{E}_{2}^{\prime}$ are $\sigma$-algebras, (i) $A \in \mathcal{A}$ implies $A^{c} \in \mathcal{A}$ and (ii) $\Omega \in \mathcal{A}$ and $\pi(\Omega)=\pi_{1}^{\prime}(\Omega)=1$. Hence, to complete the proof, we need only show that if $A^{i} \in \mathcal{A}$ for all $i=1, \ldots$ and $A^{i} \cap A^{j}=\emptyset$ for $i \neq j$, then $A:=\bigcup A^{i} \in \mathcal{A}$ and $\pi(A)=\sum_{i} \pi\left(A^{i}\right)$.

Let $N_{1}=\left\{i \mid A^{i} \in \mathcal{E}_{1}^{\prime}\right\}$ and $N_{2}=\left\{i \mid A^{i} \in \mathcal{E}_{2}^{\prime}\right\}$. Since the sets $A^{i}$ are pairwise disjoint, (1) above implies that if $i \in N_{2}$ and $\pi_{2}^{\prime}\left(A^{i}\right)>0$, then $\pi_{1}^{\prime}\left(A^{j}\right)=0$ for all $j \in N_{1}$. Then, 
(2) ensures that we must have either $A^{i} \in \mathcal{E}_{1}^{\prime}$ for all $i$ or $A^{i} \in \mathcal{E}_{2}^{\prime}$ for all $i$. Without loss of generality, assume the former. Since $\pi_{1}^{\prime}$ is prior, $A \in \mathcal{E}_{1}^{\prime} \subset \mathcal{A}$ and $\pi(A)=\pi_{1}^{\prime}(A)=$ $\sum_{i} \pi_{1}^{\prime}\left(A^{i}\right)=\sum_{i} \pi\left(A^{i}\right)$ as desired.

We have shown that $(\mathcal{A}, \pi)$ is a $\lambda$-prior. Note that $\mu_{*}(A)=(\pi(A))^{2}$ and hence, part (i) of Proposition $2 \mathrm{w},(\mathcal{A}, \pi)$ is a source with power series $\delta^{2}$. To complete the proof, we will show that $\pi$ is a Zhang source: if not, there is a source $(\mathcal{B}, \hat{\pi})$ such that $\mathcal{A} \subset \mathcal{B}$ and $\hat{\pi}(A)=\pi(A)$ for all $A \in \mathcal{A}$. Clearly, $\delta^{2}$ is the power series of $\hat{\pi}$. Let $C=[l,(m+l) / 2]$, $A=f_{1}^{-1}(C), B=f_{2}^{-1}(C)$ and $F=[A \cap B] \cup\left[A^{c} \cap B^{c}\right]$. Then, $F^{c}=\left[A \cap B^{c}\right] \cup\left[A^{c} \cap B\right]$ and $F, F^{c} \in \hat{\mathcal{E}}$. By construction,

$$
\mu_{*}(F)=\mu_{*}\left(F^{c}\right)=2 \mu\left(\bigcap_{i=1}^{4} g_{i}^{-1}(C)\right)=2\left(\frac{1}{2}\right)^{4}=\frac{1}{8}
$$

Hence, $\pi(F)=\pi\left(F^{c}\right)=1 / \sqrt{8}$, a contradiction.

\subsection{Proof of Proposition 5}

$W=(\alpha, v)$ and let $y>x$. Without loss of generality, (i.e., if necessary, by applying a positive affine transformation to $v$ ) let $v(y)=1, v(x)=0$ for the remainder of this proof.

First, let $\alpha=1$ and choose $t \in[0,1], A \in \mathcal{E}_{\pi}$, and $B \in \mathcal{E}_{\pi^{o}}$ such that $t=\pi(A)=\pi^{o}(B)$. Then, $W(y A x)=\gamma(t)$ and $W(y B x)=\gamma^{o}(t)$ and, therefore, $W(y A x) \geq W(y B x)$ if and only if $\gamma(t) \geq \gamma^{o}(t)$.

Next, let $\alpha<1, \gamma=\gamma_{\pi}$ and choose $n$ such that $(1-\alpha) n \geq 2$. Then, let $\hat{\gamma}$ be such that $\hat{\gamma}(t)=(\gamma(t))^{n}$. It is easy to show that $\hat{\gamma} \in \Gamma$. Let $\pi^{o}$ be a source such that $\gamma_{\pi^{o}} \leq \hat{\gamma}$ and let $A \in \mathcal{E}_{\pi}, B \in \mathcal{E}_{\pi^{o}}$ with $\pi^{o}(B)=\pi(A)=t$. Define $r:[0,1] \rightarrow[0,1]$ and $w:[0,1] \rightarrow[0,1]$ such that

$$
\begin{aligned}
r(t) & =(1-\alpha)(1-\hat{\gamma}(1-t)) \\
w(t) & =\alpha t+(1-\alpha)(1-\gamma(1-t))
\end{aligned}
$$

Then, for $A \in \mathcal{E}_{\pi}, B \in \mathcal{E}_{\pi^{o}}$ such that $\pi(A)=\pi^{o}(B)=t$ we have

$$
\begin{aligned}
& W(y B x)=\alpha \gamma_{\pi^{o}}(t)+(1-\alpha)\left(1-\gamma_{\pi^{o}}(1-t)\right) \geq r(t) \\
& W(y A x)=\alpha \gamma_{\pi}(t)+(1-\alpha)\left(1-\gamma_{\pi}(1-t)\right) \leq w(t)
\end{aligned}
$$


To prove the lemma, it suffices to show that $\lim _{t \rightarrow 0}(w(t) / r(t))<1$. It is easy to verify that $\gamma$ is differentiable for all $t<1$ and that $\gamma^{\prime}(t) \geq 1$ for $t \in[1-\epsilon, 1)$ for some $\epsilon>0$. Therefore, using l'Hopital's rule, we obtain:

$$
\lim _{t \rightarrow 0} \frac{\alpha t+(1-\alpha)(1-\gamma(1-t))}{(1-\alpha)(1-\hat{\gamma}(1-t))}=\lim _{t \rightarrow 1} \frac{\alpha+(1-\alpha) \gamma^{\prime}(t)}{(1-\alpha) n\left(\gamma^{\prime}(t)\right)^{n-1}} \leq \frac{\alpha+1-\alpha}{(1-\alpha) n} \leq 1 / 2
$$

\subsection{Proof of Proposition 6}

Let $W=(\alpha, v)$ and let $x_{1}<x_{2}<\ldots<x_{n}$ be the prizes in the support of $f \in \mathcal{F}_{\pi}, \pi \in$ $\Sigma$. By Lemma 2,

$$
\begin{aligned}
W(f) & =\alpha\left(v\left(x_{1}\right)+\sum_{i=2}^{n}\left(v\left(x_{i}\right)-v\left(x_{i-1}\right) \mu_{*}\left(\left\{f \geq x_{i}\right\}\right)\right)\right. \\
& +(1-\alpha)\left(v\left(x_{1}\right)+\sum_{i=2}^{n}\left(v\left(x_{i}\right)-v\left(x_{i-1}\right)\left(1-\mu_{*}\left(\left\{f \geq x_{i}\right\}\right)\right)\right.\right.
\end{aligned}
$$

Proposition 2 implies that

$$
\mu_{*}\left(\left\{f \geq x_{i+1}\right\}\right)=\gamma\left(\pi\left(\left\{f \geq x_{i+1}\right\}\right)\right)=1-F^{\gamma}\left(x_{i}\right)
$$

and, therefore,

$$
v\left(x_{1}\right)+\sum_{i=2}^{n}\left(v\left(x_{i}\right)-v\left(x_{i-1}\right) \mu_{*}\left(\left\{f \geq x_{i}\right\}\right)=\int v d F^{\gamma}\right.
$$

Similarly,

$$
1-\mu_{*}\left(\left\{f \leq x_{i}\right\}\right)=\hat{\gamma}\left(\pi\left(\left\{f \geq x_{i+1}\right\}\right)\right)=1-F^{\hat{\gamma}}\left(x_{i}\right)
$$

and, therefore,

$$
v\left(x_{1}\right)+\sum_{i=2}^{n}\left(v\left(x_{i}\right)-v\left(x_{i-1}\right)\left(1-\mu_{*}\left(\left\{f \leq x_{i}\right\}\right)\right)=\int v d F^{\hat{\gamma}}\right.
$$

The Proposition now follows since $\alpha \int v d F^{\gamma}+(1-\alpha) \int v d F^{\hat{\gamma}}=\int v d F^{\alpha \gamma+(1-\alpha) \hat{\gamma}}$. 


\subsection{Proof of Proposition 7}

Chew, Karni and Safra (1987) show that $V^{\tau v}$ is more risk averse than $V^{\bar{\tau} \bar{v}}$ if and only if $\tau \circ \bar{\tau}^{-1}$ is convex and $v \circ \bar{v}^{-1}$ is concave. If $\tau \circ \bar{\tau}^{-1}$ is twice differentiable, it is convex if and only if $\tau^{\prime \prime} \bar{\tau}^{\prime} \geq \bar{\tau}^{\prime \prime} \tau^{\prime}$. Note that $\gamma$ is twice differentiable for all $t \in[0,1)$. Substituting $\alpha \gamma+(1-\alpha) \hat{\gamma}$ for $\tau$ and $\bar{\alpha} \gamma+(1-\bar{\alpha}) \hat{\gamma}$ for $\bar{\tau}$, a straightforward calculation shows that this is true if $\alpha \geq \bar{\alpha}$.

\subsection{Proof of Proposition 8}

First, we will show that (i) implies (iii). If $v$ is not concave then $W$ is not risk averse for $\pi \in \Sigma$ with $\gamma_{\pi}=\delta^{1}$. So, assume $v$ is concave and $\alpha<1$. Fix $y>x$ and choose $1>t>\alpha$ such that $\alpha v(x)+(1-\alpha) v(y)>v(t x+(1-t) y)$. Since $v$ is continuous and concave such a $t$ exists. Let $F$ be the lottery that yields $x$ with probability $t$ and $y$ with probability $1-t$ and let $z=t x+(1-t) y$. Then, $V^{\tau_{n} v}(F)>v(z)$ for $\tau_{n}=\alpha \delta^{n}+(1-\alpha) \hat{\delta}^{n}$ and $n$ sufficiently large. Hence, $W$ is not risk averse in every source.

Next, we will prove (iii) implies (ii). Note that if $\alpha=1$ then, by Lemma $2, W(f)$ is the Choquet integral of $v \circ f$ with respect to the capacity $\mu_{*}$. Choose $E_{A}, E_{B} \in \mathcal{E}_{\mu}$ such that $E_{A} \subset A, E_{B} \subset B, \mu_{*}(A)=\mu\left(E_{A}\right)$ and $\mu_{*}(A)=\mu\left(E_{B}\right)$. Hence, $\mu_{*}(A)+\mu_{*}(B)=$ $\mu\left(E_{A}\right)+\mu\left(E_{B}\right)=\mu\left(E_{A} \cap E_{B}\right)+\mu\left(E_{A} \cup E_{B}\right) \leq \mu_{*}(A \cap B)+\mu_{*}(A \cup B)$. That is, $\mu_{*}$ is convex.

Suppose $W(g) \geq W(f)$ and let $h(\omega)=v^{-1}(\beta v(f(\omega))+(1-\beta) v(g(\omega)))$ for all $\omega \in \Omega$. Then, the characterization of uncertainty aversion in Schmeidler (1989) (i.e., the proposition on page 582) and the convexity of $\mu_{*}$ ensure that

$$
W(h)=\int v \circ h d \mu_{*}=\int(\beta v \circ f+(1-\beta) v \circ g) d \mu_{*} \geq W(f)
$$

where the integrals above are Choquet integrals. The concavity of $v$ ensures that $W(\beta f+$ $(1-\beta) g) \geq W(h)$ and therefore, $W(\beta f+(1-\beta) g) \geq W(f)$ as desired.

To prove (ii) implies (i), consider any source $\pi \in \Sigma$. Then, $f, g \in \mathcal{F}_{\pi}$ implies $\beta f+$ $(1-\beta) g \in \mathcal{F}_{\pi}$. Theorem 5 of Ergin and Gul (2009) establishes that $[f \sim g$ implies $W(\beta f+(1-\beta) g) \geq W(f)]$ is equivalent $W$ being risk averse in $\mathcal{F}_{\pi}$. Hence, (ii) implies that the source utility is risk averse in source $\pi$. Since $\pi$ was arbitrary, it follows that $W$ is risk averse in every source. 


\section{References}

Abdellaoui, M., (2000) "Parameter-Free Elicitation of Utility and Probability Weighting Functions," Management Science, 46, 11, 1497-1512.

Abdellaoui, M., A. Baillon, L. Placido, and P. P. Wakker, (2011) "The Rich Domain of Uncertainty: Source Functions and Their Experimental Implementation," American Economic Review, 101, 3,695-723.

Allais, M., 1953, Le comportement de l'homme rationnel devant le risque: critique des postulats et axiomes de l'ecole Amiricaine, Econometrica 21, 503-546.

Billingsley, P. (1995), Probability and Measure. 3rd Edition. New York: John Wiley \& Sons.

Camerer, C. F., and T. H. Ho, (1994), "Violations of the betweenness axiom and nonlinearity in probability," Journal of Risk and Uncertainty, 8, 167-196.

Camerer, C. and M. Weber, (1992), "Recent developments in modeling preferences: Uncertainty and ambiguity," Journal of Risk and Uncertainty, 5, pp. 325-70.

Chew, S. H., L. Epstein, and U. Segal, (1991), "Mixture Symmetry and Quadratic Utility," Econometrica, Vol. 59, No. 1 (Jan., 1991), pp. 139-163.

Chew, S. H., E. Karni, and Z. Safra, (1987), "Risk Aversion in the Theory of Expected Utility with Rank Dependent Probabilities," Journal of Economic Theory, 42(2), 370-381.

Chew S. H., and J. Sagi, (2008) "Small worlds: Modeling attitudes toward sources of uncertainty," Journal of Economic Theory, 139, Issue 1, pp. 1-24.

Chipman, J. S. (1960). "Stochastic Choice and Subjective Probability." In D. Willner (ed.), Decisions, Values and Groups, Volume I. Oxford, England: Pergamon Press, pp. 70-95.

Choquet, G. (1953-54). "Theory of Capacities," Annales de l'lnstitut Fourier

Curley, S. E and F. J. Yates. (1989): "An Empirical Evaluation of Descriptive Models of Ambiguity Reactions in Choice Situations," Journal of Mathematical Psychology 33, pp. 397-427.

Dave C., C. C. Eckel, C. A. Johnson, C. (2010): "Eliciting risk preferences: When is simple better?" Journal of Risk and Uncertainty, December 2010, 41, Issue 3, pp 219-243.

Einav, I., A. Finkelstein, I. Pascu, and M. R. Cullen (2012): "How General Are Risk Preferences? Choices under Uncertainty in Different Domains," American Economic Review 2012, 102(6): 26062638.

Ellsberg, D. (1961): "Risk, Ambiguity and the Savage Axioms," Quarterly Journal of Economics, 75, 643-669. 
Epstein L. G. and J. Miao, (2003) "A Two-Person Dynamic Equilibrium under Ambiguity, Journal of Economic Dynamics and Control 27 (2003).

Epstein, L. G, (2001) "Sharing Ambiguity, American Economic Review 91 (2001), 45-50.

Epstein and Wang, T. (1994) 'Intertemporal Asset Pricing under Knightian Uncertainty," Econometrica, Vol. 62, 2, pp. 283-322.

Ergin, H. and F. Gul (2009): "A theory of subjective compound lotteries," Journal of Economic Theory 144, 3, 899-929.

Fox, C. R. and A. Tversky, (1995): "Ambiguity Aversion and Comparative Ignorance," Quarterly Journal of Economics, 110, 3, 585-603.

French, K. R., and J. M. Poterba, (1991): "Investor diversification and international equity markets," American Economic Review 81, 222-226.

Gilboa, I. and M. Marinacci, (2013), "Ambiguity and the Bayesian Paradigm," in: Advances in Economics and Econometrics, Tenth World Congress (ed. Acemoglu D, Arellano M and E. Dekel), Volume 1, Chapter 7, pp 179-243.

Ghirardato, P. (2010), "Ambiguity," Encyclopedia of Quantitative Finance.

Ghirardato, P. and M. Marinacci (2002), "Ambiguity Made Precise: A Comparative Foundation," Journal of Economic Theory, 102 (2), pp. 251-289.

Grubel (1968), "Internationally Diversified Portfolios: Welfare Gains and Capital Flows," American Economic Review , 58, 5 , 1299-1314.

Gul, F., and W. Pesendorfer (2012): "Expected Uncertain Utility Theory," mimeo. Princeton University.

Heath, C. and A. Tversky, (1991): "Preference and Belief: Ambiguity and Competence in Choice under Uncertainty," Journal of Risk and Uncertainty, 4, 5-28.

Hurwicz, L. (1951): "Some Specification Problems and Applications to Econometric Methods (Abstract)," Econometrica, 19, 343-344.

Isaac, R. M. and D. James (2000): "Just Who Are You Calling Risk Averse?" Journal of Risk and Uncertainty, 20(2), 177-187.

Keynes, John Maynard. (1921). A Treatise on Probability. London: Macmillan.

Klibanoff, P., M. Marinacci and S. Mukerji (2005): "A smooth model of decision making under ambiguity," Econometrica 73(6) (November, 2005) pp. 1849-1892.

Lease, R., W. Lewellen, and G. Schlarbaum (1974): "Individual Investor Attributes and Attitudes," Journal of Finance, May 1974, 29, 413-33.

Machina, M. J. (1982): “'Expected Utility' Analysis without the Independence Axiom," Econometrica, 50, 2, 277-323. 
Machina, M. J. (1987): "Choice Under Uncertainty: Problems Solved and Unsolved," Journal of Economic Perspectives, 1, 121-154.

Machina, M. J. (2009): "Event Separability in the Ellsberg Urn," mimeo. UCSD.

Machina, M. J. and D. Schmeidler (1992): "A More Robust Definition of Subjective Probability," Econometrica, 60, 745-780.

Nau, R.F. (2006): "Uncertainty Aversion With Second-Order Utilities and Probabilities," Management Science, 52:1, 136-145

Prelec, D. (1998): "The Probability Weighting Function," Econometrica, 66 (3), 497-527.

Quiggin, J. (1982): "A Theory of Anticipated Utility," Journal of Economic Behavior \& Organization, 3, Issue 4, 323-343.

Savage, L. J. (1954): The Foundations of Statistics, Wiley, New York.

Segal, U. (1990): "First Order versus Second Order Risk Aversion," Journal of Economic Theory, 51(1), 111-125.

Schmeidler, D. (1989): "Subjective Probability and Expected Utility without Additivity," Econometrica, Vol. 57, 3, 571-587.

Starmer, C. (2000): "Developments in Non-Expected Utility Theory: The Hunt for a Descriptive Theory of Choice under Risk," Journal of Economic Literature, XXXVIII (June 2000), 332-382.

Strassen, V. (1964). "Messfehler und Information," Zeitschrift fuer Wahrscheinlichkeitstheory und Verwandte Gebiete, 2, 273-305.

Tversky, A. and C. R. Fox, "Weighing Risk and Uncertainty," Psychological Review, CXLIX (1995),269-83.

Tversky, A., Kahneman, D. (1992): "Advances in prospect theory: cumulative representation of uncertainty," Journal of Risk and Uncertainty, 5, 297-323.

Tversky A. and P. Wakker (1995): "Risk Attitudes and Decision Weights," Econometrica, 63,6, pp. 1255-1280.

van der Waart, A. W. and J. A. Wellner (1996), "Weak Convergence and Empirical Processes," New York: Springer Verlag.

Wu and Gonzalez, R. (1996). Curvature of the probability weighting function. Management Science, 42, 1676-1690.

Zhang, J., (2002): "Subjective Ambiguity, Expected Utility and Choquet Expected Utility," Economic Theory, 20, 159-181. 